\title{
Structural basis for activation and non- canonical catalysis of the Rap GTPase activating protein domain of plexin
}

\author{
Yuxiao Wang ${ }^{1 \dagger}$, Heath G Pascoe ${ }^{1 \dagger}$, Chad A Brautigam², Huawei He ${ }^{1 \ddagger}$, Xuewu Zhang ${ }^{1,2 \star}$ \\ ${ }^{1}$ Department of Pharmacology, University of Texas Southwestern Medical Center, \\ Dallas, United States; ${ }^{2}$ Department of Biophysics, University of Texas Southwestern \\ Medical Center, Dallas, United States
}

*For correspondence: xuewu. zhang@utsouthwestern.edu

†These authors contributed equally to this work

¥Present address: State Key Laboratory of Silkworm Genome Biology, Southwest University, Chongqing, China

Competing interests: The authors declare that no competing interests exist.

Funding: See page 23

Received: 24 July 2013

Accepted: 22 August 2013

Published: 01 October 2013

Reviewing editor: Axel T Brunger, Howard Hughes Medical Institute, Stanford University, United States

(c) Copyright Wang et al. This article is distributed under the terms of the Creative Commons Attribution License, which permits unrestricted use and redistribution provided that the original author and source are credited.
Abstract Plexins are cell surface receptors that bind semaphorins and transduce signals for regulating neuronal axon guidance and other processes. Plexin signaling depends on their cytoplasmic GTPase activating protein (GAP) domain, which specifically inactivates the Ras homolog Rap through an ill-defined non-canonical catalytic mechanism. The plexin GAP is activated by semaphorin-induced dimerization, the structural basis for which remained unknown. Here we present the crystal structures of the active dimer of zebrafish PlexinC1 cytoplasmic region in the apo state and in complex with Rap. The structures show that the dimerization induces a large-scale conformational change in plexin, which opens the GAP active site to allow Rap binding. Plexin stabilizes the switch II region of Rap in an unprecedented conformation, bringing Gln63 in Rap into the active site for catalyzing GTP hydrolysis. The structures also explain the unique Rap-specificity of plexins. Mutational analyses support that these mechanisms underlie plexin activation and signaling.

DOI: 10.7554/eLife.01279.001

\section{Introduction}

Plexins are a large group of type I transmembrane proteins that serve as the major receptors for semaphorins (Yazdani and Terman, 2006; Tran et al., 2007). Plexin-mediated semaphorin signaling controls neuronal axon guidance as well other essential processes such as angiogenesis and immune responses (Sakurai et al., 2012; Takamatsu and Kumanogoh, 2012). Aberrant plexin/semaphorin signaling has been implicated in numerous pathologies including neurological disorders and cancer (Yaron and Zheng, 2007; Tamagnone, 2012; Gu and Giraudo, 2013). Plexins all possess a large multidomain extracellular region, a single transmembrane helix and a multi-domain cytoplasmic region. Binding of semaphorin to the extracellular region of plexin triggers activation of the cytoplasmic region, which relays the signal to downstream pathways.

The plexin cytoplasmic region contains a juxtamembrane segment, a RhoGTPase binding domain (RBD) and a GTPase activating protein (GAP) domain (Rohm et alo, 2000; Hu et alo, 2001; He et alo, 2009; Tong et al., 2009; Bell et al., 2011). The juxtamembrane segment has been suggested to regulate plexin signaling by interacting with the GAP domain or mediating oligomerization (He et al., 2009; Bell et al., 2011). Binding of RhoGTPases such as Rac1 and RND1 to the RBD facilitates plexin activation (Vikis et al., 2000; Driessens et al., 2001; Zanata et al., 2002; Turner et al., 2004; Tong et al., 2007), the mechanism of which is not well understood (Bell et al., 2011; Wang et al., 2012). The GAP domain in plexin shows structural homology to RasGAPs such as p120GAP, and contains a functionally essential arginine residue corresponding to the catalytic 'arginine finger' in RasGAPs (Rohm et al., 2000; Oinuma et al., 2004; He et al., 2009). Plexins have been reported previously to be GAPs for the Ras homologs R-Ras and M-Ras (Oinuma et al., 2004; Saito et al., 2009). Our recent study, however, has demonstrated that the plexin GAP does not act directly on R-Ras or M-Ras (Wang et al., 2012). Instead 
eLife digest A key question in neurobiology is how the brain becomes wired up. How do axons-the 'wires' along which neural signals flow-know in which direction to grow to reach their intended targets? A family of signalling proteins called semaphorins contribute to this process by acting as stop signals for axons that are heading in the wrong direction. The actions of semaphorins are mediated by receptors known as plexins, which are found on the membranes of axons.

Plexins contain an extracellular domain that binds semaphorin, and a large domain inside the cell that can turn semaphorin binding into cellular responses. When a semaphorin protein binds to the extracellular domain of a plexin receptor, the domain inside the cell joins with the intracellular domain of a neighbouring receptor to form a dimer. This activates the intracellular domain, which turns on its ability to inactivate a molecule called Rap. The end result is that the axon stops growing and changes direction, but the molecular mechanisms through which these events occur are not well understood.

Now, Wang, Pascoe et al. have worked out the structure of the dimers formed by the intracellular plexin domains, both alone and in complex with Rap. The structures reveal how the dimer drives a shape change of the intracellular domain to enable it to bind Rap, and show that Rap itself adopts a novel conformation upon binding to plexin. This conformational change in Rap catalyses the breakdown of a signalling molecule called GTP, which inactivates Rap and triggers an intracellular signalling cascade that causes the axon to collapse and change direction.

Lastly, Wang, Pascoe et al. have shown that the highly specific nature of these interactions depends on particular amino-acid residues in both Rap and the plexin receptor. Further work is now required to determine whether this pattern of activation represents a general mechanism for signalling by plexin receptors, and for the inhibition of Rap.

DOI: 10.7554/eLife.01279.002

it is active specifically to the Ras homolog Rap, and this RapGAP activity is critical for plexin signaling. GTP-bound active Rap is a key activator of integrin for promoting cell-matrix adhesion (Gloerich and Bos, 2011). Conversion of Rap into the GDP-bound inactive form by the plexin GAP likely contributes to plexin-mediated repulsive axon guidance and other cell morphological changes through causing inactivation of integrin and weakening cell-matrix adhesion (Wang et al., 2012).

RasGAPs such as p120GAP and neurofibromin facilitate GTP hydrolysis of Ras, R-Ras and M-Ras by providing the conserved arginine finger to stabilize the leaving Y-phosphate group ( Li et al., 1997; Scheffzek et al., 1997; Scheffzek et al., 1998; Quilliam et al., 1999; Ohba et al., 2000; Bos et al., 2007). Concomitantly, a conserved glutamine in the GTPases (Gln61 in Ras) coordinates the nucleophilic water for hydrolysis. Many other GAP/small GTPase pairs use similar mechanisms to catalyze GTP hydrolysis (Bos et al., 2007). Rap is distinct from Ras/R-Ras/M-Ras in that it has a threonine at position 61, which lacks the ability to coordinate the catalytic water. Canonical RapGAPs are structurally unrelated to RasGAPs and catalyze Rap GTP hydrolysis by providing an asparagine residue (referred to as the 'Asn thumb') to fulfill the water coordination role of Gln61 in Ras (Scrima et al., 2008). SynGAP (Synaptic GAP), and three GAP1 family members Rasal (Ras-GTPase-activating-like protein), CAPRI ( $\mathrm{Ca}^{2+}$-promoted Ras inactivator) and GAP1 1P4BP (tetrakisphosphate binding protein) are dualspecificity GAPs, active to both Ras and Rap. Plexins and these dual-specificity GAPs share the RasGAP fold that contains the arginine finger but lack a conserved Asn thumb (Kupzig et al., 2006; Pena et al., 2008). They facilitate GTP hydrolysis for Rap through a distinct, poorly understood mechanism. A recent study has suggested that Gln63 in Rap plays a role analogous to Gln61 in Ras in the noncanonical catalysis of the dual-specificity GAPs (Sot et al., 2010). Mutating Gln63 in Rap abolishes GTP hydrolysis catalyzed by both the dual-specificity GAPs and plexins (Sot et al., 2010; Wang et al., 2012).

Plexin signaling is critically dependent on the on/off switch of the RapGAP activity under the control of semaphorin (Wang et al., 2012). Our previous structural analyses have suggested that the plexin GAP is autoinhibited by adopting a closed conformation that sequesters the active site (He et al., 2009). A pre-formed inhibitory dimer of plexin may also be involved in suppressing the GAP activity prior to semaphorin binding (Antipenko et al., 2003; Tong et al., 2007; Nogi et al., 2010). Semaphorins are dimeric molecules and have been suggested to induce dimerization or oligomerization 
of plexin for triggering downstream signaling (Klostermann et al., 1998; Koppel and Raper, 1998; Driessens et al., 2001; Perrot et al., 2002; Antipenko et al., 2003; Love et al., 2003). A model of plexin activation involving oligomerization mediated by the RBD/RhoGTPase interaction has been proposed (Bell et al., 2011), but existence of this oligomeric structure in solution or on the cell surface has not been established (Siebold and Jones, 2013). Recent structural studies have demonstrated how dimeric semaphorin brings two copies of the plexin extracellular region into proximity (Janssen et al., 2010; Liu et al., 2010; Nogi et al., 2010; Janssen et al., 2012). We have shown that the purified plexin cytoplasmic region displays low RapGAP activity, which can be activated dramatically by fusing it to the coiled-coil dimerization motif of GCN4 (general control non-repressed 4) (Wang et al., 2012). These observations collectively support that semaphorin-induced formation of an active dimer of plexin is the major mechanism for activation of the GAP domain and intracellular signaling.

In this study we sought to understand how the plexin RapGAP is activated by induced-dimerization and facilitates GTP hydrolysis specifically for Rap. We systematically screened various coiled-coil dimer fusions of the plexin cytoplasmic region for optimal activation of the GAP. These experiments led to crystallization and structure determination of the active dimer of zebrafish PlexinC1. In addition, we employed a novel protein ligation system to covalently link the plexin cytoplasmic region and Rap, which stabilized their weak interaction and allowed us to crystallize and determine the structure of a PlexinC1/Rap complex. The structures and the associated mutational analyses together reveal the basis for the dimerization-induced activation, the non-canonical catalysis and the unique specificity of plexin for Rap.

\section{Results and discussion}

\section{Screening and crystallization of the coiled-coil-induced active dimer of plexin $_{\text {cyto }}$}

Our previous study has shown that the RapGAP activity of the cytoplasmic region of plexins (plexins cyto) can be activated by fusing it to the coiled-coil motif of GCN4 through a flexible linker of various lengths (Wang et al., 2012). Our extensive crystallization trials of these coiled-coil induced dimers of plexins syto all failed, presumably due to the flexibility of the linker. We therefore removed the linker and directly fused the coiled-coil with the juxtamembrane helix (the $\mathrm{N}$-terminal helix in the juxtamembrane

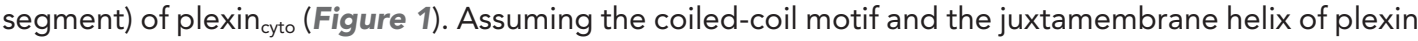
merge into a continuous helix, varying the relative register between them by adding or removing residues at the junction can result in dramatically different relative orientations between the two plexin monomers in the dimer. Without knowing the ideal arrangement of the two monomers for active dimer formation, we systematically tested fusing plexin cyto $_{\text {to }}$ each of the seven unique positions on the heptad repeat of the coiled-coil (Figure 1A). We used mouse PlexinA $1_{\text {cyto }}$ for the screening experiments, because it displayed the highest level of activation by induced dimerization in our previous study (Wang et al., 2012). We chose Ala1272, located near the N-terminus of the juxtamembrane helix in PlexinA $1_{\text {cytor }}$ as the reference for naming the fusion constructs. These constructs are referred to as $\mathrm{CC}(\mathrm{x}) \mathrm{Plexin} \mathrm{A} 1_{\text {cytor }}$ in which ' $x$ ' indicates the position of Ala1272 in PlexinA1 on the heptad repeat (Figure 1A).

GAP activity assays showed that all these dimer constructs are substantially more active than the monomer (Figure 1C). Remarkably, CC(a)PlexinA $1_{\text {cyto, }}$ CC(d)PlexinA $1_{\text {cyto }}$ and CC(g)PlexinA $1_{\text {cyto, }}$ which confer in general similar inter-monomer orientations, achieve much higher activation levels than $C C(b)$ PlexinA $1_{\text {cytor }}, C C(c) P l e x i n A 1_{\text {cytor }}, C C(e) P$ lexinA $1_{\text {cyto }}$ and $C C(f) P l e x i n A 1_{\text {cyto. }}$. We also tested four zebrafish $\mathrm{CC}(\mathrm{x}) \mathrm{Plexin} C 1_{\text {cyto }}$ constructs, which showed the same trend of activation levels (Figure $\left.1 \mathrm{~A}, \mathrm{D}\right)$. These results further support the notion that a specific association mode between the two plexin monomers is required for the optimal dimerization-induced activation (Wang et al., 2012). We screened for crystals of those highly active dimer constructs, and obtained crystals of zebrafish CC(a)PlexinC $1_{\text {cyto }}$ and determined the structure at $3.3 \AA$ resolution (Table 1).

\section{Characterization and crystallization of ligated plexin $_{\text {cyto }} /$ Rap complexes}

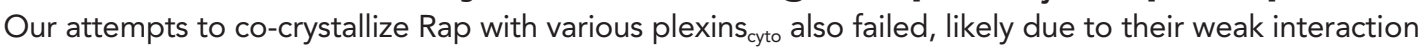
(Wang et al., 2012). To stabilize the interaction, we covalently linked plexins $\mathrm{s}_{\text {cyto }}$ and Rap1B in vitro by using a protein ligation system based on the transpeptidase activity of sortase from Staphylococcus aureus (Figure 2; see details in 'Materials and methods') (Popp et al., 2009). We used the GAP activity assay to characterize the ligated complex of zebrafish PlexinC $1_{\text {cyto }}$ and human Rap1B connected by a 
A

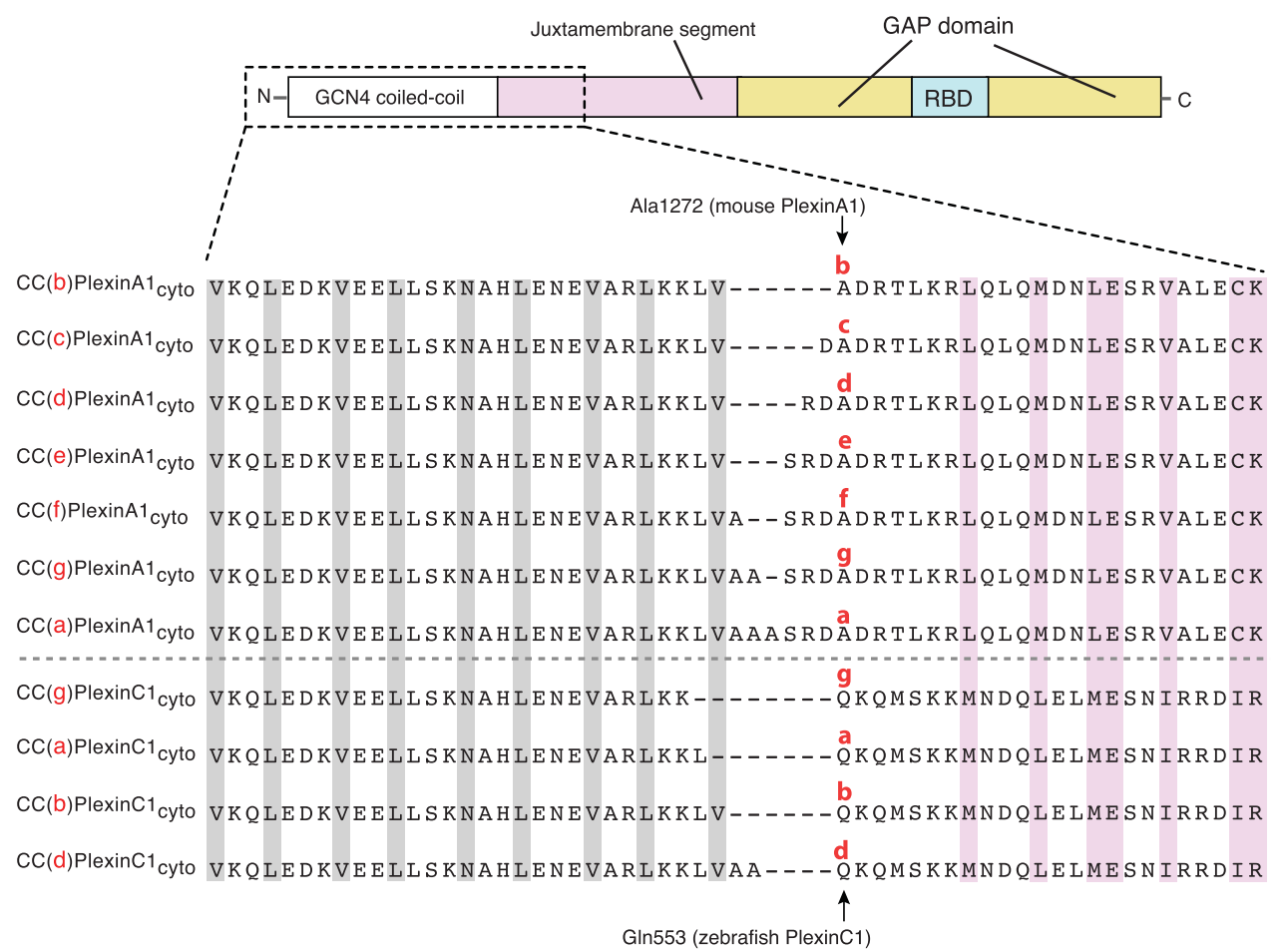

B

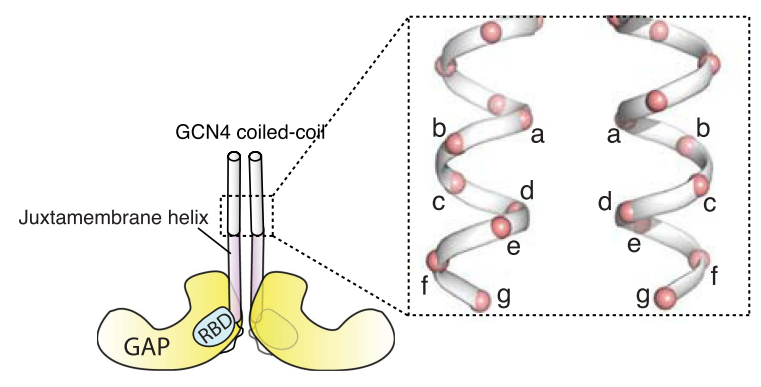

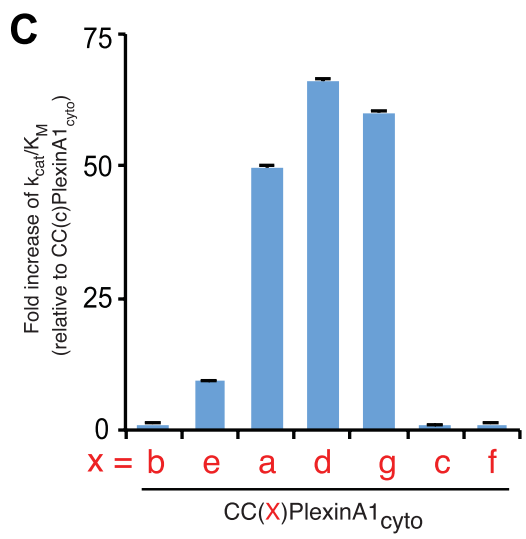

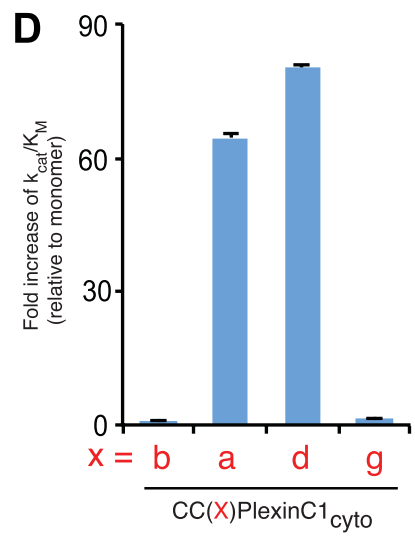

Figure 1. Activation of the plexin GAP by coiled-coil fusion. (A) Design of the coiled-coil fusions of mouse PlexinA $1_{\text {cyto }}$ and zebrafish PlexinC $1_{\text {cyto. }}$. The juxtamembrane segment sequences from mouse PlexinA $1_{\text {cyto }}$ and zebrafish PlexinC $1_{\text {cyto }}$ are aligned. The constructs are named CC(x)Plexin ${ }_{\text {cyto }}$ where ' $x$ ' (in red) is the position of Ala1272 in PlexinA1 or Gln553 in PlexinC1 on the heptad repeat. The ' $a$ ' and ' $d$ ' positions in the GCN4 coiled-coil are highlighted gray. Residues at the active dimer interface are highlighted pink. (B) Diagram of the CC(x)Plexin cyto constructs. (C) GAP activity of mouse $C C(x)$ PlexinA $1_{\text {cyto. }}$. Activity of monomeric PlexinA $1_{\text {cyto }}$ is too low to be measured reliably. The fold increase of $k_{\text {cat }} / K_{M}$ is calculated relative to $C C$ (c) PlexinA $1_{\text {cyto, }}$ which is the least active among the dimers but approximately 10 -fold more active than the monomer. (D) GAP activity of zebrafish CC( $x$ ) PlexinC $1_{\text {cyto. }}$ In both (C) and (D), error bars represent standard error of the $\mathrm{k}_{\text {cat }} / \mathrm{K}_{\mathrm{M}}$.

DOI: 10.7554/eLife.01279.003

24-residue flexible linker and the sortase-recognition motif. The ligated complex catalyzes GTP hydrolysis at much higher rates than the two individual proteins mixed at the same concentrations (Figure 2C), indicating enhanced formation of the catalytically competent plexin/Rap complex when the two proteins are tethered.

As mentioned above, the dimerization induces the active conformation of plexin cytor $_{\text {which }}$ enhances Rap binding and GTP hydrolysis. Conversely, stabilization of the active conformation of plexin by Rap binding is expected to facilitate formation of the plexin dimer. Due to basal GAP activity of plexin cytor $_{\text {Rap in the ligated plexin }}$ cyto $/$ Rap complex is GDP-bound and cannot stably 
Table 1. Data collection and refinement statistics

\begin{tabular}{|c|c|c|}
\hline \multicolumn{3}{|l|}{ Data collection } \\
\hline Crystal & CC(a)Plexin $C 1_{\text {cyto }}$ & $\begin{array}{l}\text { PlexinC1 } 1_{\text {cyto }} / \\
\text { Rap1B }\end{array}$ \\
\hline Space group & $\mathrm{P} 2{ }_{1} 2_{1} 2_{1}$ & $\mathrm{P} 1$ \\
\hline \multicolumn{3}{|l|}{ Cell dimensions } \\
\hline$a, b, c(\AA)$ & $\begin{array}{l}53.22,146.10 \\
209.58\end{array}$ & $\begin{array}{l}76.28,84.73 \\
138.75\end{array}$ \\
\hline$\alpha, \beta, \gamma\left(^{\circ}\right)$ & $90,90,90$ & $\begin{array}{l}91.09,95.15, \\
90.32\end{array}$ \\
\hline Resolution $(\AA ̊)$ & $\begin{array}{l}50.0-3.30 \\
(3.36-3.30)^{\star}\end{array}$ & $\begin{array}{l}50.0-3.30 \\
(3.36-3.30)^{\star}\end{array}$ \\
\hline$R_{\text {sym }}$ & 11.1(86.8) & $5.2(48.7)$ \\
\hline$I / \sigma$ & $19.7(1.4)$ & 18.6(1.6) \\
\hline Completeness (\%) & $95.8(79.5)$ & 91.0(89.2) \\
\hline Redundancy & $10.4(4.3)$ & $1.9(1.9)$ \\
\hline \multicolumn{3}{|l|}{ Refinement } \\
\hline Resolution (Å) & 3.30 & 3.30 \\
\hline No. reflections & 21,087 & 47,207 \\
\hline Completeness(\%) & $83.32 \dagger$ & 90.23 \\
\hline$R_{\text {work }} / R_{\text {free }}(\%)$ & $22.6 / 28.2$ & $24.3 / 30.0$ \\
\hline No. atoms & 8888 & 22,228 \\
\hline Protein & 8871 & 22,086 \\
\hline Ligand/ion & 0 & 132 \\
\hline Water & 17 & 10 \\
\hline \multicolumn{3}{|l|}{ B-factors } \\
\hline Protein & 98.9 & 143.5 \\
\hline Ligand/ion & - & 128.2 \\
\hline Water & 49.3 & 89.7 \\
\hline \multicolumn{3}{|l|}{ R.m.s deviations } \\
\hline Bond lengths ( $(\AA)$ & 0.005 & 0.004 \\
\hline Bond angles $\left({ }^{\circ}\right)$ & 0.85 & 0.70 \\
\hline \multicolumn{3}{|l|}{ Ramanchandran plot } \\
\hline Favored (\%) & 91.7 & 93.1 \\
\hline Allowed (\%) & 8.1 & 6.7 \\
\hline Disallowed (\%) & 0.2 & 0.2 \\
\hline
\end{tabular}

*Highest resolution shell is shown in parenthesis.

†The data were corrected for anisotropy in HKL2000. This treatment eliminated many weak reflections and reduced the completeness of the data used for refinement compared to the completeness reported for data collection.

DOI: 10.7554/eLife.01279.004 brane helix does not mediate any inter-molecular
interactions and likely has some flexibility. The flexibility can further compensate for deletion or insertion of one residue at the junction between the coiled-coil and the juxtamembrane helix, allowing several constructs to induce the active dimer and achieve similarly high activation levels (Figure 1). More deletions or insertions at the junction probably cannot be accommodated without severe distortion of the juxtamembrane helix, explaining the much lower activation levels of those constructs (Figure 1).

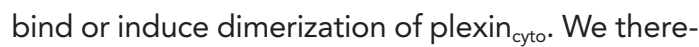
fore used the $Y$-phosphate analog aluminum fluoride $\left(\mathrm{AlF}_{\mathrm{x}, \mathrm{x}}=3\right.$ or 4 ) (Vetter and Wittinghofer, 2001) to induce formation of the transition state complex between Rap(GDP) and plexin. Our analytical ultracentrifugation experiments showed that while the ligated PlexinC $1_{\text {cyto }} /$ Rap1B complex itself did not dimerize, it dimerized robustly in the presence of $A F_{x}$ (Figure 2D). We crystallized this complex with $\mathrm{AlF}_{\mathrm{x}}$ and determined the structure to $3.3 \AA$ resolution (Table 1).

\section{Overall structures of the $\mathrm{CC}(\mathrm{a})$ PlexinC $1_{\text {cyto }}$ dimer and the PlexinC $1_{\text {cyto }} /$ Rap complex}

In the CC(a)PlexinC $1_{\text {cyto }}$ structure, the two plexin monomers in the asymmetric unit form a symmetric side-by-side dimer (Figure 3A). The two juxtamembrane helices are oriented approximately in parallel, extending well beyond the main body of the proteins and integrating into the C-termini of the coiled-coil moiety. On the plasma membrane, this configuration of the plexin dimer orients the active sites of the two GAP domains toward the membrane surface and leaves sufficient space for binding of the membrane anchored Rap substrate, as observed in the PlexinC $1_{\text {cyto }}$ / Rap1B complex structure (Figure $3 A, B$ ).

The asymmetric unit of the PlexinC $1_{\text {cyto }} /$ Rap $1 B$ complex structure contains four protomers of the complex, which are virtually identical to one another. The four PlexinC1 molecules form two pairs of dimers, consistent with the dimerization observed in solution. The conformation of PlexinC1 and its mode of dimerization are highly similar in the two structures (Figure 3C), supporting that they represent the active state of plexin and are not artifacts induced by the fusion constructs.

The coiled-coil moiety in the CC(a)PlexinC $1_{\text {cyto }}$ structure is nearly identical to the isolated coiledcoil structures reported previously (O'Shea et al., 1991). Comparison of the active dimers in the two structures suggests that there is a small geometric incompatibility between the coiled-coil and the plexin dimer, as the $\mathrm{N}$-terminal portion of the juxtamembrane helix (residues 553-566) seems to bend slightly near its junction with the coiledcoil (Figure $3 \mathrm{C}$ ). This portion of the juxtamembrane helix does not mediate any inter-molecular 
A

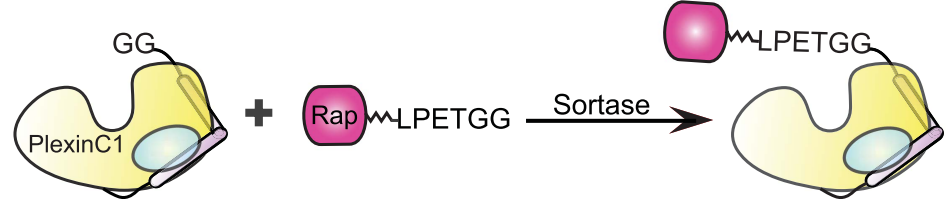

B

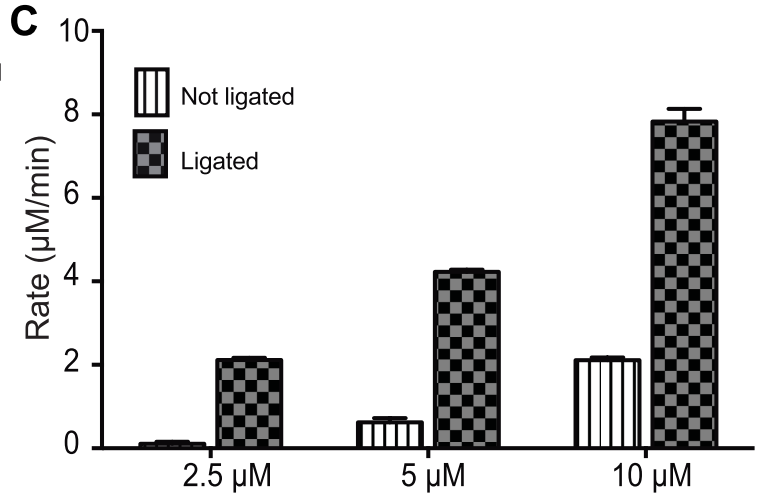

D
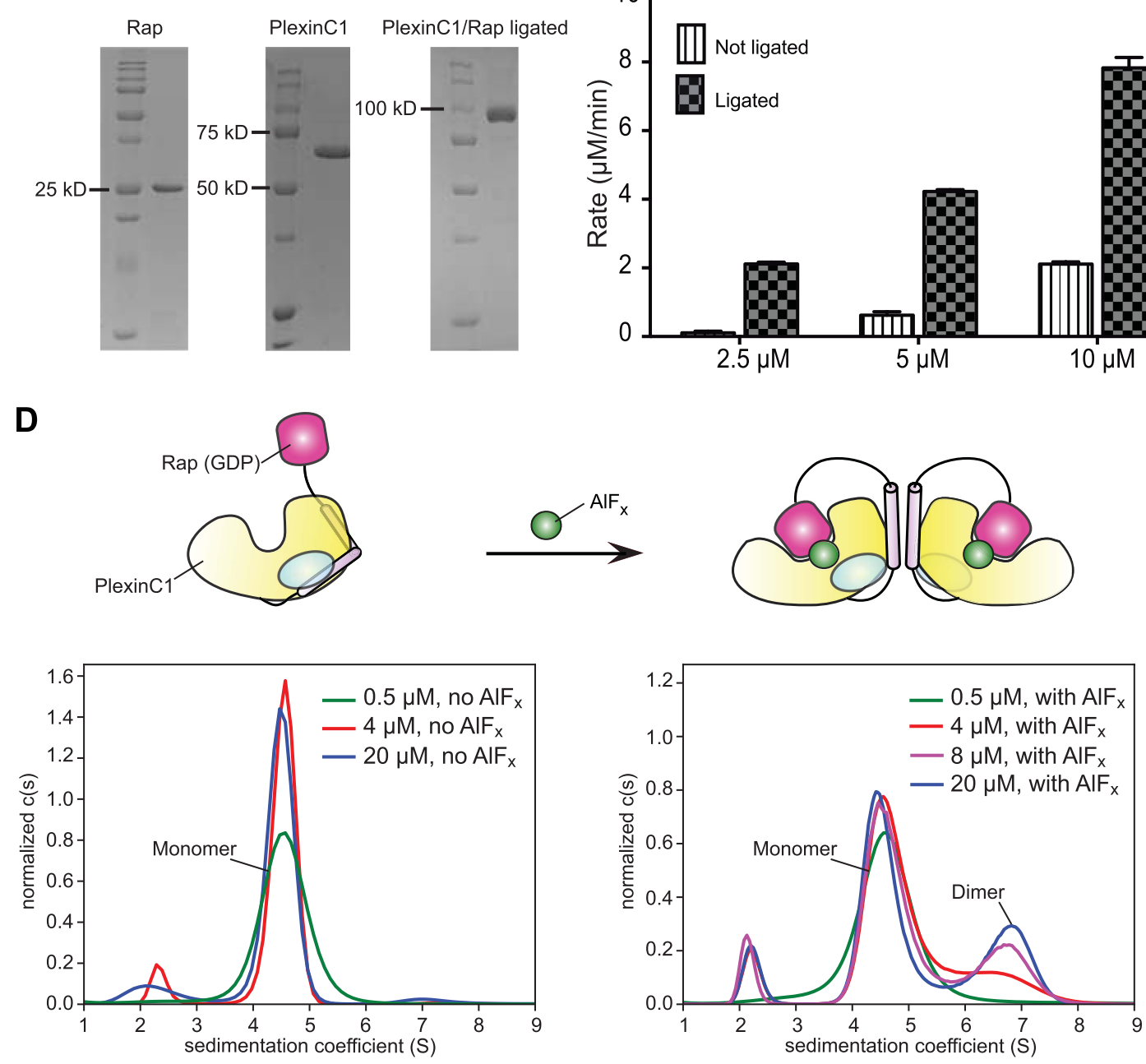

Figure 2. Sortase-mediated ligation and characterization of the ligated Plexin $C 1_{\text {cyto }} /$ Rap1B complex. (A) Scheme of the sortase-mediated ligation. (B) Representative gels of purified PlexinC1 cyto, Rap1B and the ligated PlexinC1 cyto/Rap1B complex with the 24-residue linker and the 'LPETGG' sortase recognition motif. (C) Comparison of the GTP hydrolysis activity between the ligated complex and the individual PlexinC1 $1_{\text {cyto }}$ and Rap1B proteins mixed at the same concentrations. The hydrolysis rates are averages of three replicates. Error bars represent standard deviation of the mean. (D) Analytical ultracentrifugation showing $\mathrm{AlF}_{\mathrm{x}}$-induced dimerization of the ligated PlexinC $1_{\text {cyto }} /$ Rap $1 \mathrm{~B}$ complex. In the absence of $\mathrm{AlF}$ (the left panel), the majority of the complex behaves as a monomer with a sedimentation coefficient of $4.5 \mathrm{~S}$. In the presence of $\mathrm{AlF}_{\mathrm{x}}$ (the right panel), a dimeric species (sedimentation coefficient of $6.7 \mathrm{~S}$ ) appears and becomes more abundant at higher protein concentrations.

DOI: 10.7554/eLife.01279.005

\section{Interactions in the dimer interface}

We will refer to the $\mathrm{CC}(\mathrm{a})$ Plexin $\mathrm{1}_{\text {cyto }}$ structure for the following discussion on the active dimer unless otherwise stated, because the dimer interface in this structure is better resolved in the electron density map. The dimer interface is formed by the juxtamembrane helix and one side of the GAP domain, burying a total of $\sim 3200 \AA^{2}$ surface area (Figure 4). The RBDs in the two monomers are far away from each other and not involved in dimer formation. The center of the dimer interface is a 4-helix bundle 


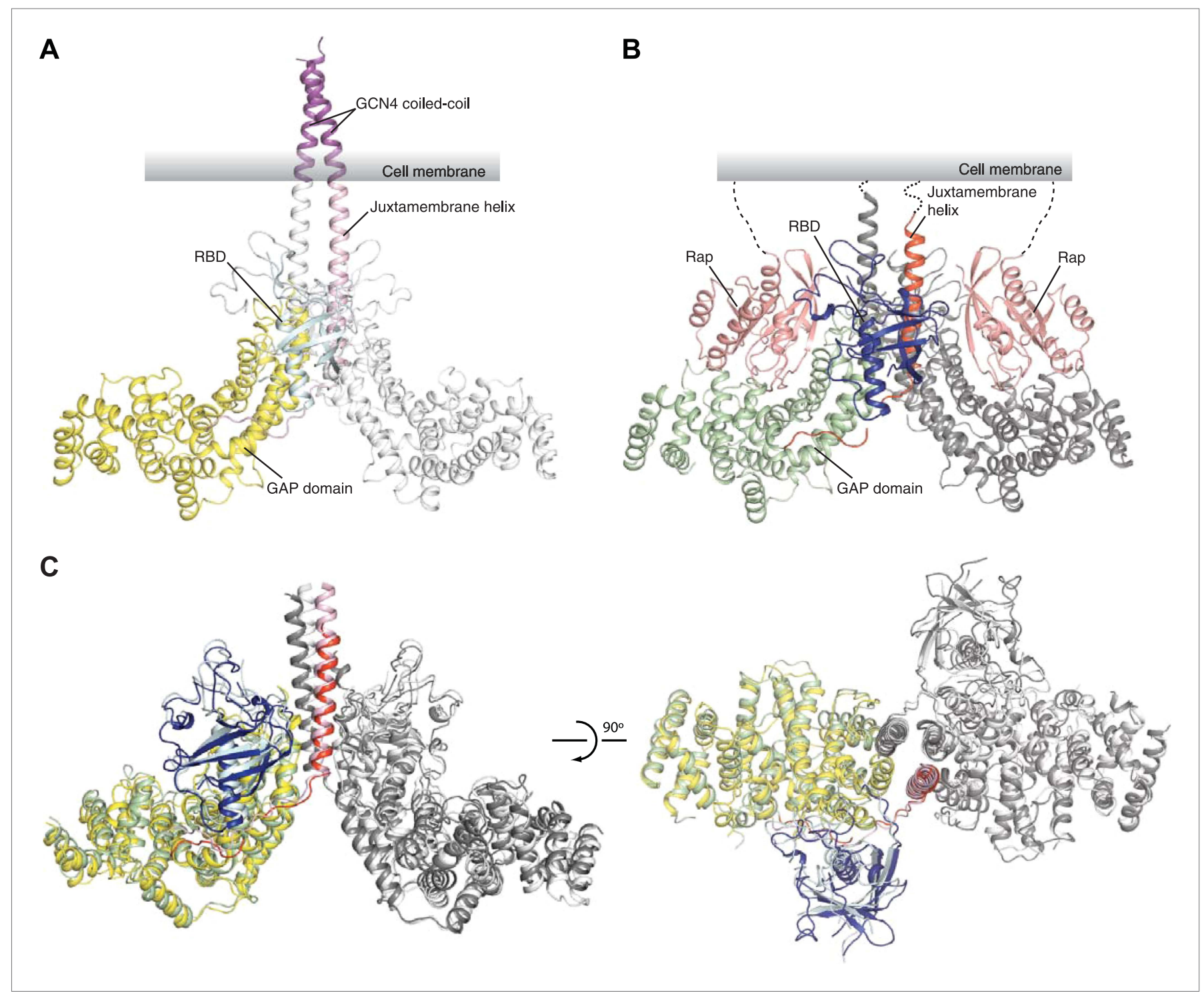

Figure 3. Overall structures of the zebrafish CC(a)PlexinC1 $1_{\text {cyto }}$ active dimer and the PlexinC $1_{\text {cyto }} /$ Rap $1 \mathrm{~B}$ complex. (A) Structure of the $\mathrm{CC}(\mathrm{a}) \mathrm{PlexinC} 1_{\text {cyto }}$ dimer. (B) Structure of the PlexinC1 cyto $/$ Rap1B complex. One of the two active dimers of plexin with Rap1B bound in the asymmetric unit is shown. In both (A) and (B), domains from one plexin monomer in the dimer are colored and labeled. The other monomer is shown in white in (A) and gray in (B). (C) Comparison of the active dimers in the structures of CC(a)PlexinC $1_{\text {cyto }}$ and the PlexinC $1_{\text {cyto }} /$ Rap $1 \mathrm{~B}$ complex. The coiled-coil moiety is omitted for clarity. The color schemes are the same as in (A) and (B).

structure comprised of the C-terminal portion of the juxtamembrane helix (residues 567-584) and the $\mathrm{N}$-terminal portion of helix 11 in the GAP domain (residues 929-943) from each monomer (Figure 4). The core of the 4-helix bundle is dominated by hydrophobic interactions, involving residues Ile571, Ile575, Phe579 and Leu582 from the juxtamembrane helix and Met933, Ile936 and Leu939 from helix 11 (Figure 4B). The core interface is supported by peripheral electrostatic interactions mediated by Arg572, Arg576 and Asp581 from the juxtamembrane helix and Glu770, Glu932, and Lys937 from the GAP domain (Figure 4A).

A loop-helix segment (residues 1038-1058) between helix 15 and 17 in the GAP domain of each monomer wraps around the C-terminal portion of the 4-helix bundle. The interactions involve Leu1045, Lys1047, Leu1054, Leu1055, and Lys1058 in the loop-helix segment and Phe579, Gln583, Thr584 and Leu939 from the 4-helix bundle (Figure 4A). We call the loop-helix element 'the activation segment' in plexin since it plays a major role in regulating the GAP activity (see the next section for details), functionally resembling the well known activation segment in protein kinases (Huse and Kuriyan, 2002). 


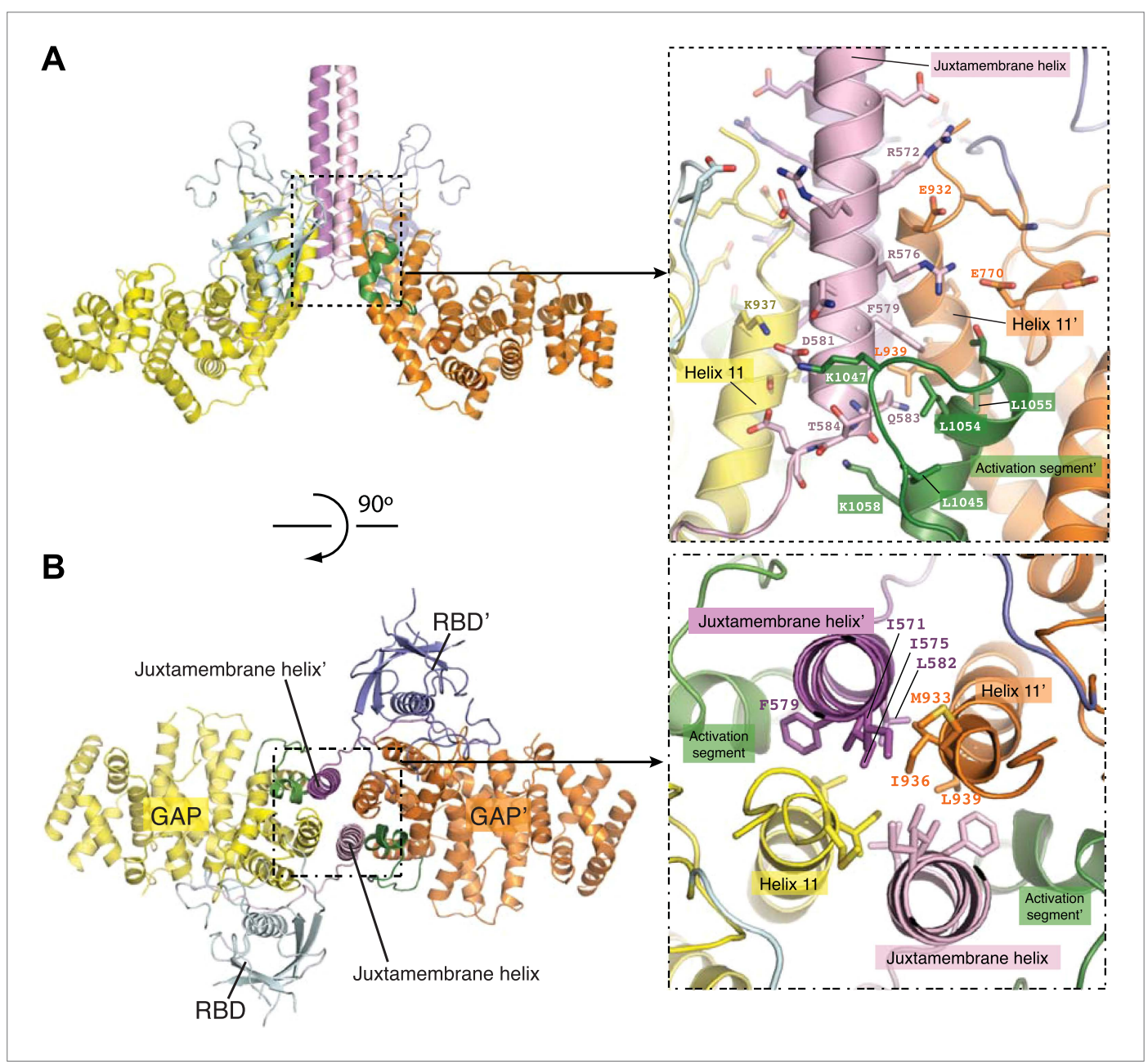

Figure 4. The dimer interface in the $C C(a) P l e x i n C 1_{\text {cyto }}$ structure. (A) Periphery of the dimer interface. The coiledcoil moiety is not shown. (B) Hydrophobic core of the dimer interface. Residue labels for one monomer are omitted for clarity.

DOI: 10.7554/eLife.01279.007

\section{Dimerization induced conformational changes that lead to GAP activation}

Comparisons of the dimer structure with previously determined structures of plexins cyto reveal several substantial conformational differences. The most striking difference is in the juxtamembrane helix (Figure 5A). Except in one of the PlexinB1 structures where it is disordered (Bell et alo, 2011), the juxtamembrane helix in all other previous structures adopts a kinked conformation, with both the $\mathrm{N}$ and C-terminal halves interacting with the GAP domain (He et al., 2009; Tong et al., 2009; Wang et al., 2012). In the active dimer structure, the last two turns in the juxtamembrane helix (residues 585591 , corresponding to residues 1282-1288 in mouse PlexinA3) convert to an extended loop. This loop and the following segment use Asp588, Leu589, Asp591 and Val593 to make a distinct set of intra-molecular interactions with the GAP domain (Figure 5B). The remaining $\mathrm{N}$-terminal helical portion (residues 553-584) adopts a straight conformation and rotates by $\sim 90^{\circ}$ in relation to the inactive structures (Figure 5A) to mediate the formation of the 4-helix bundle at the center of the dimer interface (Figure 4B). Helix 11 undergoes a small tilt to accommodate the juxtamembrane helix from the dimer partner, and the top part (residues 929-934) adopts a $3_{10}$ helix like conformation to pack against the hydrophobic core of the 4-helix bundle (Figure $5 \mathrm{C}$ ).

The conformational changes in the juxtamembrane helix and helix 11 are coupled to changes in the activation segment. In all the previously reported structures of plexins cyto, the highly conserved helical portion of the activation segment adopts essentially the same 'closed' conformation (Figure 6A) (He et al., 2009; Tong et al., 2009; Bell et al., 2011; Wang et al., 2012). An asparagine residue in the 


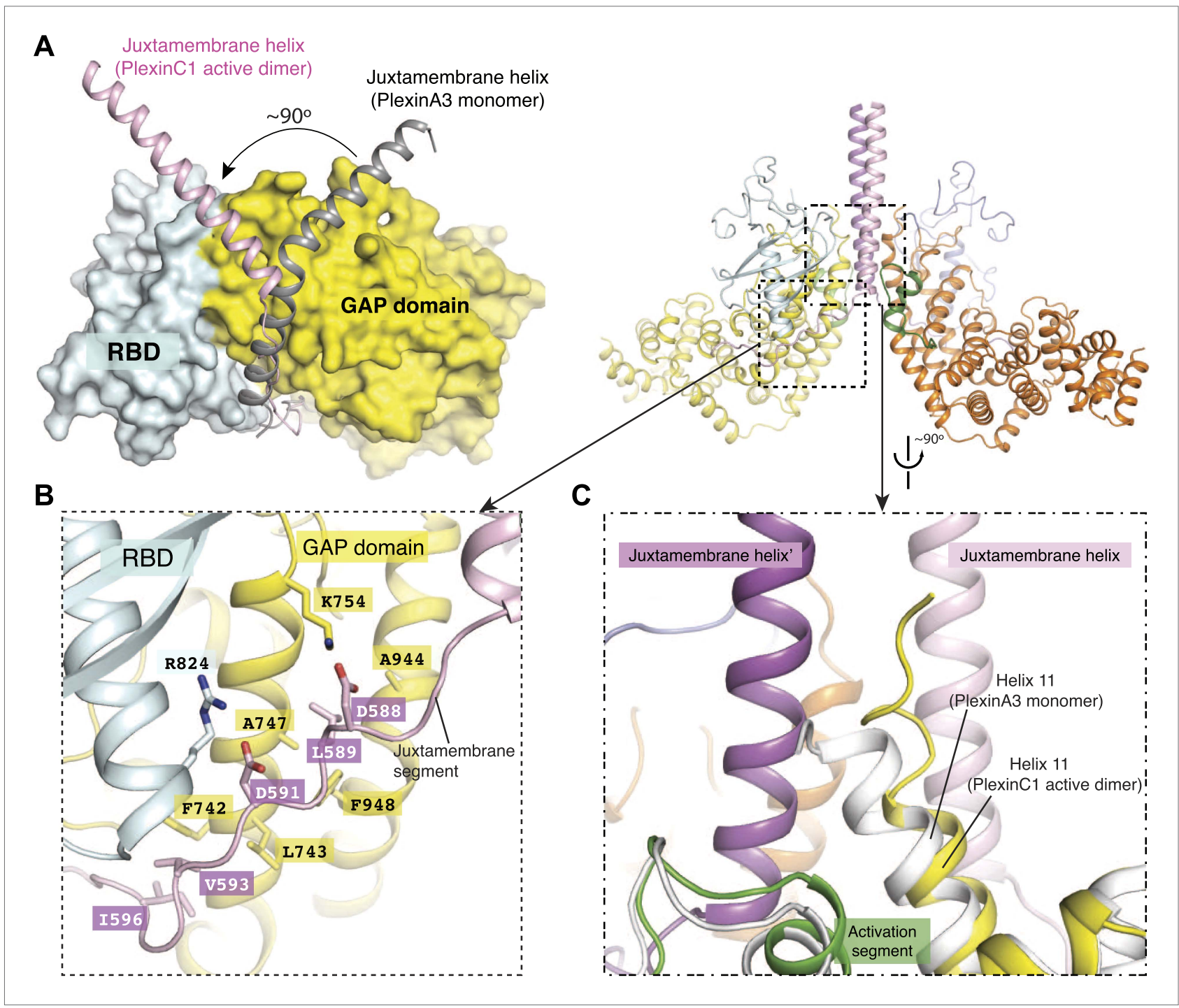

Figure 5. Dimerization-induced conformational changes of the juxtamembrane helix and helix 11. (A) Conformational change of the juxtamembrane helix. One monomer in the PlexinC $1_{\text {cyto }}$ active dimer is superimposed onto the monomeric PlexinA $3_{\text {cyto }}$ structure (PDB code: 3 IG3). The GAP domain and RBD of Plexin $\mathrm{A}_{\text {cyto }}$ are shown in the surface representation. (B) Intra-molecular interactions made by the extended portion of the juxtamembrane segment in the CC(a)PlexinC1 $1_{\text {cyto }}$ structure. (C) Conformational change of helix 11. The structure superimposition is the same as in (A).

DOI: 10.7554/eLife.01279.008

helix (Asn1774 in mouse PlexinA3) is invariably hydrogen bonded with a conserved aspartate (Asp1758 in PlexinA3) in helix 15. A proline residue (Pro1772 in PlexinA3) at the N-terminus of the helix acts as a lid that covers the asparagine and blocks its access to the incoming Rap substrate. Docking Rap to PlexinA3 based on the PlexinC1/Rap complex structure results in a number of clashes between Rap and the activation segment (Figure 6A). The proline 'lid' (Pro1772) sterically clashes with Tyr40 in Rap, while the carbonyl oxygen on the sidechain of Asn1774 makes an unfavorable contact with the sidechain of Asp38 in Rap. The loop portion of the activation segment appears to be rather flexible, as it displays high B-factors in PlexinA3 (PDB ID: 3IG3) and the PlexinB1/Rac1 complex (PDB ID: 3SU8) and is partially disordered in apo-PlexinB1 (PDB ID: 3HM6) and the PlexinA1/Rac1 complex (PDB ID: 3RYT). The loop likely samples many conformations, some of which may impose additional hindrance on Rap binding.

In contrast, the activation segment in the active dimer adopts an open conformation and shifts away from the GAP active site (Figure $6 B, C$ ). This shift appears to be induced by the interactions between the activation segment and the 4-helix bundle in the dimer interface (Figure 6C). The outward 
A

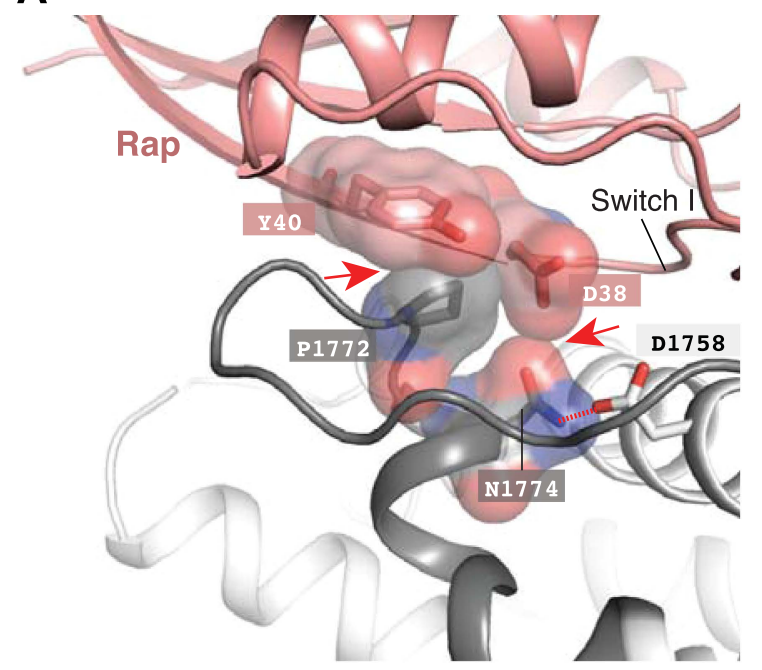

PlexinA3 monomer $\left\{\begin{array}{l}\bigcirc \text { Activation segment } \\ \bigcirc \text { GAP domain }\end{array}\right.$

C

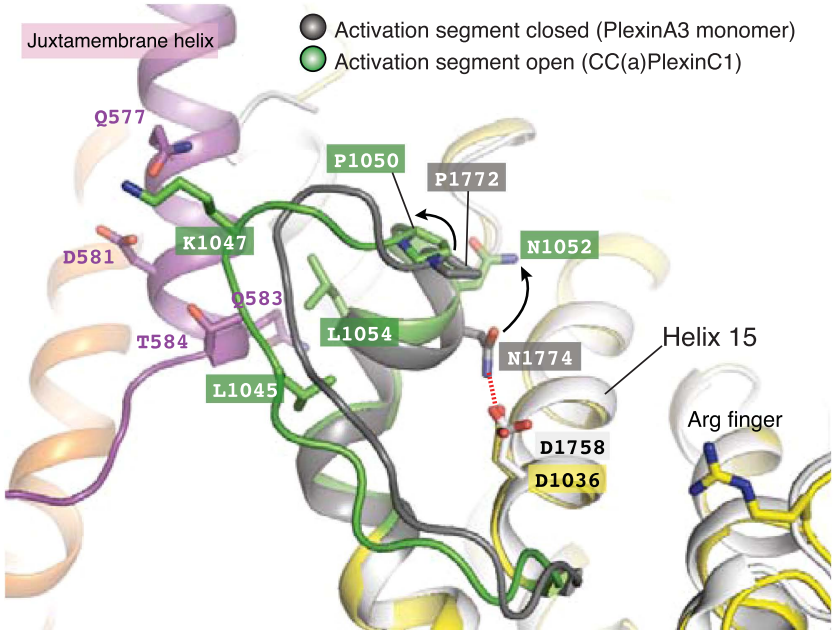

B

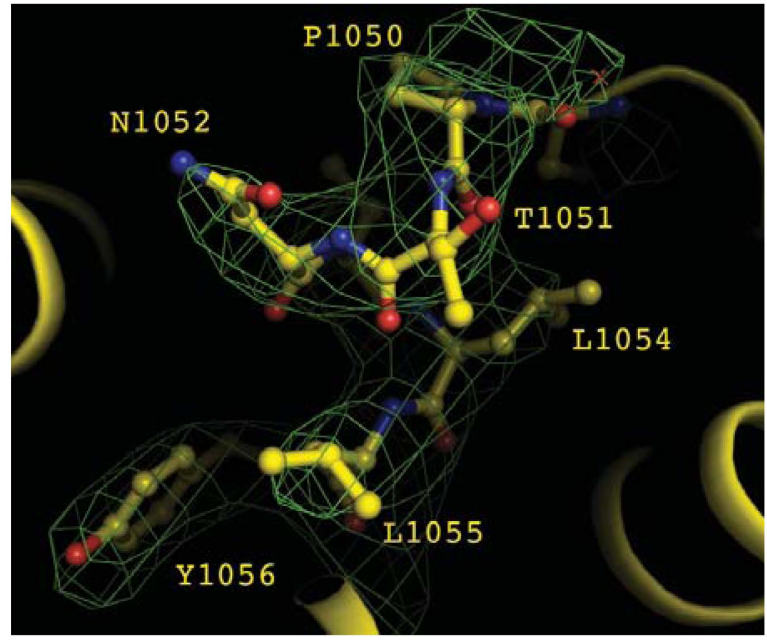

D

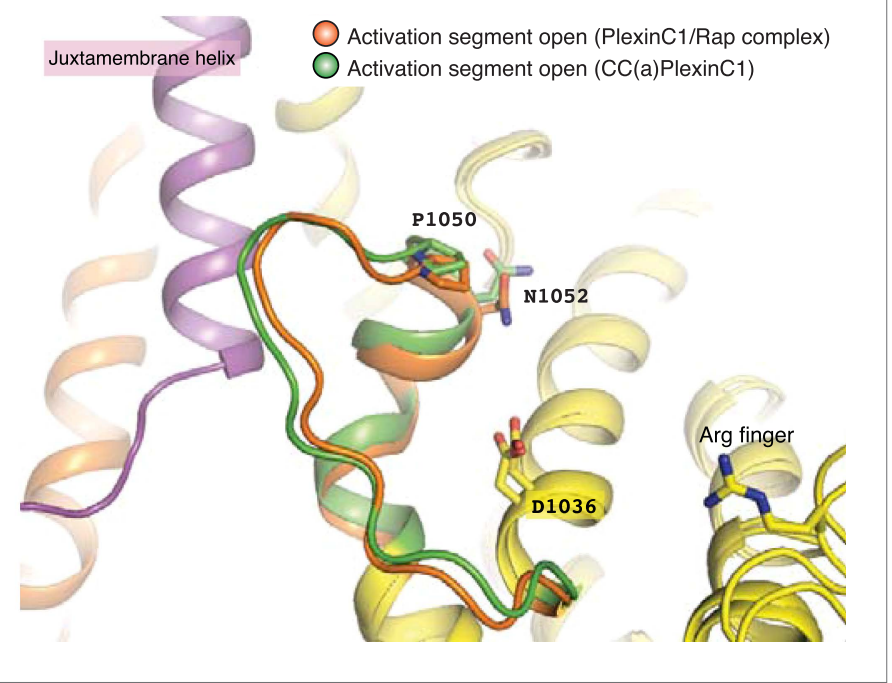

Figure 6. Dimerization-induced opening of the activation segment. (A) Docking of Rap to the inactive PlexinA3 cyto structure (PDB code: 31 G3). The docking is based on a superimposition between PlexinA3 and PlexinC1 in the PlexinC1/Rap complex structure (see 'Materials and methods' for details). Red dashed line: hydrogen bond. Red arrows: steric clashes and unfavorable interactions. (B) Sigma-A weighted simulated annealing omit map of the activation segment in CC(a)PlexinC1 cyto. The map was calculated using the model with residues 1050-1056 in one of PlexinC1 molecules removed. The map was contoured at $3 \sigma$, with the final model shown. (C) Comparison of the activation segment in the structures of CC(a)PlexinC $1_{\text {cyto }}$ and PlexinA $3_{\text {cyto. }}$. Conformational differences important for GAP activation are highlighted by black arrows. (D) Comparison of the activation segment in the structures of CC(a)PlexinC1 $1_{\text {cyto }}$ and the PlexinC1 $1_{\text {cyto }} /$ Rap complex.

DOI: 10.7554/eLife.01279.009

shift pulls Asn1052 (Asn1774 in PlexinA3) away from Asp1036 (Asp1758 in PlexinA3), precluding hydrogen bond formation. Pro1050 in the dimer structure also moves outward compared to Pro1772 in PlexinA3 (Figure 6C). The activation segment in the structure of the PlexinC1/Rap complex adopts a similar open conformation (Figure 6D). Therefore, a major mechanism in the dimerization-induced activation of plexin appears to be the outward shift of the activation segment, which opens the otherwise obstructed active site to allow Rap binding and catalysis of GTP hydrolysis. While this conformational change in the plexin GAP domain seems small, it is known that interactions between small GTPases and their regulators or effectors can be strongly influenced by subtle changes at the binding interface 
(Nassar et al., 1996; Snyder et al., 2002). The activation segment in the PlexinC1/Rap complex is slightly more closed than that in the coiled-coil-induced PlexinC1 dimer (Figure 6D), indicating that the active dimer promotes a conformation that is more open than required for accommodating Rap. Binding of Rap induces a slight closure of the active site for optimal interactions and catalysis of GTP hydrolysis.

The RBD and the subdomain composed of the first three and the last two helices in the GAP domain show conformational variations among all the structures of plexins. Given the fact that they are not involved in the dimer interface or Rap binding, the variations of these structural elements likely reflect their intrinsic flexibility and are not relevant to the activation mechanism.

\section{Mutational analysis of the active dimer structure}

We performed extensive mutational analyses to test the activation mechanism revealed by the dimer structure. Arg576, Asp581, Asp588, Val593 and Met933 are involved in the dimer interface or intramolecular interactions that stabilize the new conformation of the juxtamembrane segment (Figures 4 and 5B). In the inactive monomer structures, residues at these positions are surface exposed and do not make any interactions. We made the R576E, D581K, D588K, V593E and M933E single mutations

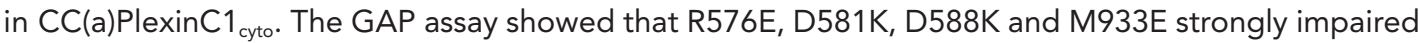
dimerization-induced activation (Figure 7A). The deleterious effect of V593E on GAP activation is weaker but clearly observable at a lower plexin concentration (Figure 7A, C). To test the coupling between the dimerization and the conformation of the activation segment, we designed the Q583A, T584A, L1045A, K1047A and L1054A mutations to disrupt the interactions between the activation segment and the juxtamembrane helix from the dimer partner (Figure 6C). The GAP assay showed that while K1047A modestly decreased dimerization-induced GAP activation, L1054A, L1045A and T584A greatly reduced the activation (Figure 7B,C).

We further examined the activation mechanism by using a functional assay, which assesses the ability of plexin to induce COS7 cell collapse upon semaphorin stimulation (Takahashi et al., 1999). Since the ligand for zebrafish PlexinC1 was not available, mouse PlexinA3 and its ligand Sema3F were used in these assays (He et al., 2009). The K1273E, E1278R, E1285R and M1290E mutations of mouse PlexinA3, corresponding to R576E, D581K, D588K and V593E of zebrafish PlexinC1 respectively, all significantly impaired plexin-mediated COS7 cell collapse (Figure 7D). A previous study identified a large panel of mutations that abolished PlexinB1-mediated COS7 cell collapse (Bell et al., 2011). These mutations were designed to test the model of plexin activation by Rac1-induced oligomerization. The results are also consistent with the activation mechanism shown here, as most of the mutated resides are conserved in zebrafish PlexinC1 and are involved in formation of the active dimer. Some mutations of highly conserved residues in the dimer interface have been identified in cancer patients, including R2040W in PlexinB1 (Gui et al., 2011) (corresponding to Lys1058 in zebrafish PlexinC1) and R1680Q/W in PlexinA2 (Cancer Genome Atlas Research Network, 2012) (corresponding to Lys937 in zebrafish PlexinC1). Both of these mutations likely prevent formation of the active dimer of plexins, consistent with the tumor suppressor function of plexins suggested by previous studies (Gu and Giraudo, 2013).

\section{Overall binding mode between zebrafish PlexinC $1_{\text {cyto }}$ and human Rap1B}

The species mismatch of the plexin/Rap complex does not affect their interaction, since the human Rap1B construct contains only three residues non-identical to their counterparts in zebrafish Rap1B, which are all located far from the plexin/Rap interface (Figure $\mathbf{8 A}$, middle panel). The linker between PlexinC $1_{\text {cyto }}$ and Rap1B is not visible in the electron density map, suggesting that it is flexible as designed and does not impose restraints on the plexin/Rap interaction. A superimposition of Rap and Ras in the PlexinC $1_{\text {cyto }} /$ Rap and p120GAP/Ras complexes shows that the overall binding modes of the two with their respective GAPs are similar (Figure 8A, left panel) (Scheffzek et al., 1997). The GAP domain in PlexinC1 and Switches I (residues 30-38) and II (residues 59-67) in Rap constitute the majority of the binding interface, whereas the RBD in plexin is not involved and its role in GAP regulation remains unclear (Figure 8). The core of the interface is composed of several hydrophobic residues, which are surrounded by numerous charge-charge interactions at the periphery. Most of the Rap-binding residues are conserved among the plexin family members, suggesting that they all interact with Rap in the same mode (Figure 8B,C). The presumed arginine finger (Arg711) in PlexinC1 superimposes well with the arginine finger (Arg789) in p120GAP, playing the same role in catalysis by interacting with the $A_{1} F_{x}$ and GDP in the active site (Figure $8 A$ ). While the bound $A F_{x}$ is not clearly resolved in the relatively 

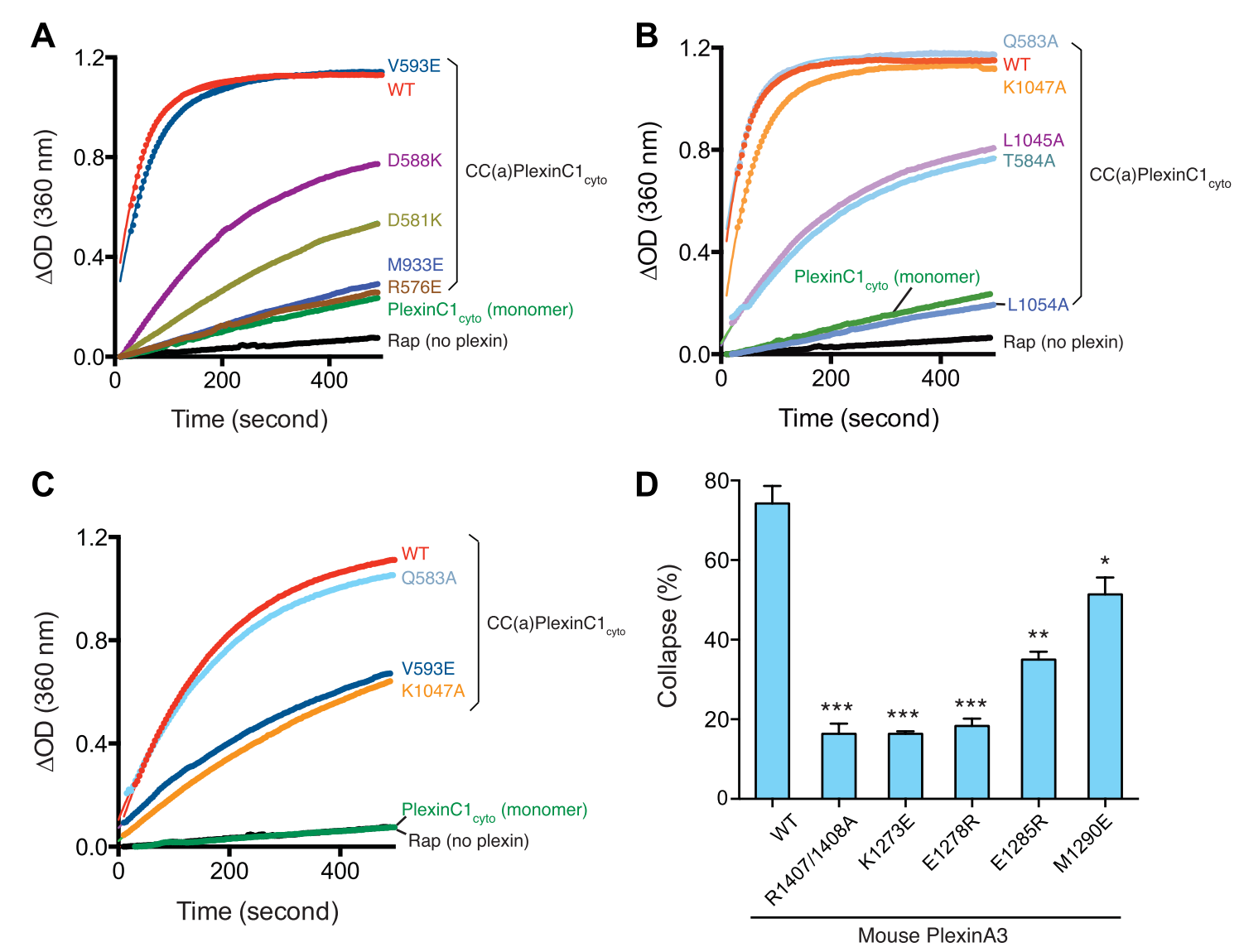

Figure 7. Mutational analyses of the dimerization-driven activation mechanism. (A-C) Mutational analyses of the activation mechanism using the GAP activity assay. Residues mutated in (A) are involved in stabilizing the active dimer, whereas residues in (B) couple the dimer formation to the opening of the activation segment. In (A) and (B) the concentration of plexin is $2 \mu \mathrm{M}$. In (C), the concentration of plexin is $0.25 \mu \mathrm{M}$. The Rap concentration is $120 \mu \mathrm{M}$ for all the assays. Data shown are representative of three replicates. (D) Mutational analyses using the COS7 cell collapse assay. The results for the wild type and the arginine-finger mutant (R1407/1408A) are shown as positive and negative controls, respectively. Error bars represent standard error of the mean from three independent experiments. At least 150 cells were counted for each sample in each experiment. Statistical significance between wild type and each mutant is determined by two-tailed Student's t-test $\left({ }^{\star} \mathrm{p}<0.05 ;{ }^{* \star} \mathrm{p}<0.01 ;{ }^{\star \star \star} \mathrm{p}<0.001\right)$.

low-resolution map, the shape of the density suggests that it is the trigonal $\mathrm{AlF}_{3}$, the same as in the p120GAP/Ras structure. We therefore modeled it $\mathrm{AlF}_{3}$ in the structure. The second conserved arginine (Arg1001) in PlexinC1 is equivalent to Arg903 in p120GAP, which stabilizes the position of the arginine finger (Figure 8A). The functional importance of these two arginine residues in plexin has been demonstrated by previous mutational studies (Rohm et al., 2000; Oinuma et al., 2004; He et al., 2009; Wang et al., 2012).

\section{Interaction between the activation segment in plexin and the Switch I of Rap}

Switch I of Rap makes numerous interactions with the activation segment in PlexinC1 (Figure 9A). As mentioned above, the activation segment in the PlexinC1/Rap complex structure adopts the open conformation similar to that in the CC(a)PlexinC1 dimer structure. Pro1050 at the N-terminus of the helical portion of the activation segment packs against Tyr40 in Rap. Asn1052 forms two hydrogen bonds with the carboxyl group of Asp38 and the backbone amide of Ser39 in Rap. Lys 1053 apparently makes electrostatic interactions with Asp38 in Rap and Asp1036 in plexin. Gln1032 in helix 15 also contributes to Switch I binding through forming three hydrogen bonds. GAP activity assays showed that while the P1050A mutation caused a modest activity decrease, the Q1032E, N1052E and K1053A mutations largely abolished the activity (Figure 9B). COS7 cell collapse assays showed that both the 


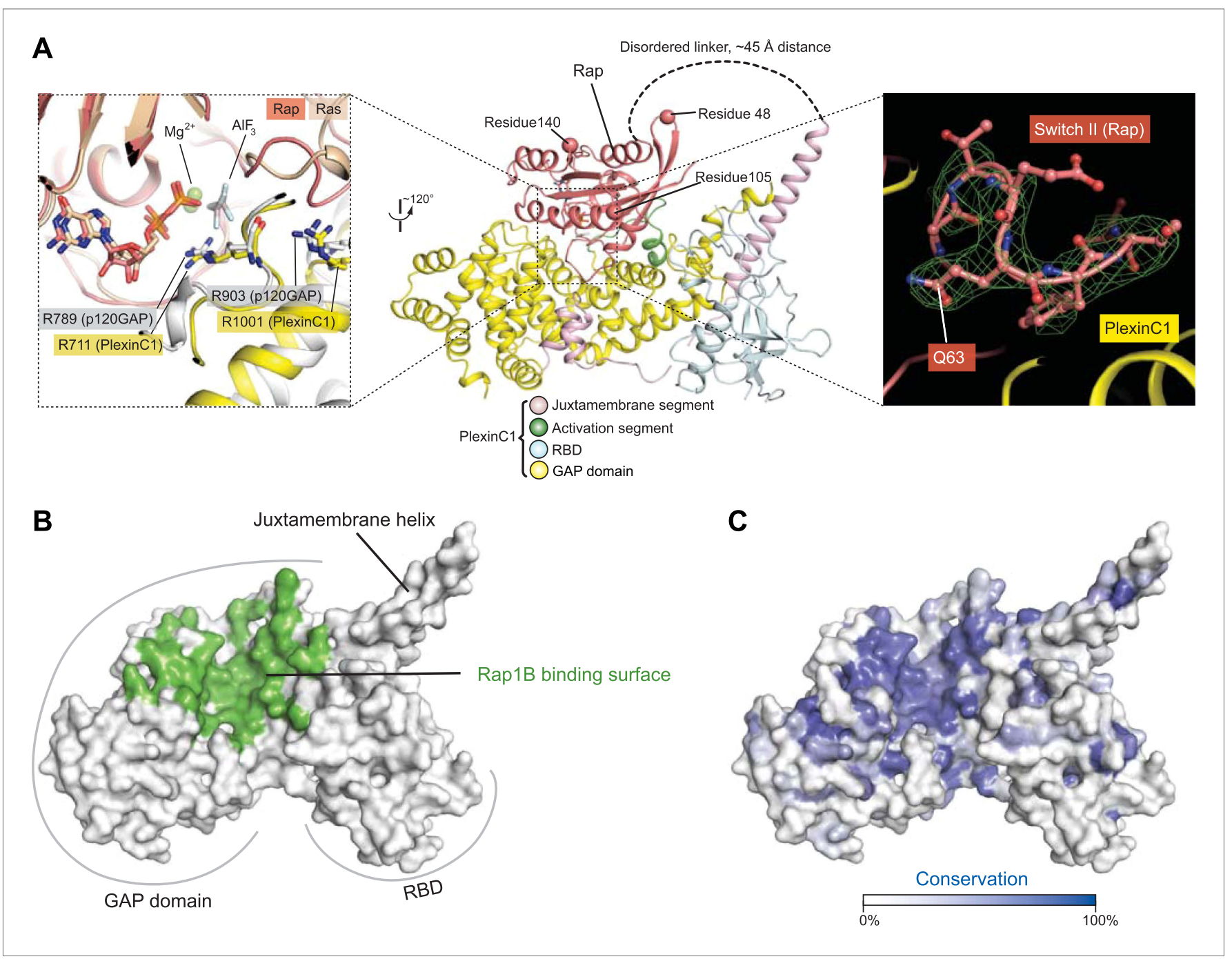

Figure 8. Overall view of the interface between zebrafish PlexinC $1_{\text {cyto }}$ and human Rap1B in the complex structure. (A) The PlexinC $1_{\text {cyto }} /$ Rap interface and its comparison with that in the p120GAP/Ras complex structure. The middle panel shows the overall structure of the PlexinC1 cyto/Rap complex, with the three residues $(48,105$ and 140) different between human and zebrafish Rap1B highlighted. The left panel shows a superimposition of the active sites in

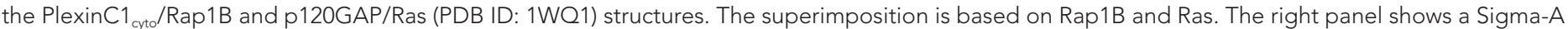
weighted simulated annealing omit map of Switch II in Rap, calculated using the model with residues 60-66 in one of the Rap1B molecules removed. The map is contoured at $3 \sigma$, with the final model of the structure shown. (B) Rap-binding surface on PlexinC1 cyto. Residues in PlexinC1 within $4 \AA$ distance of the bound Rap1B molecule are colored green. (C) Sequence conservation projection on the molecular surface of PlexinC1 $1_{\text {cyto }}$. The conservation scores were calculated based on an alignment of zebrafish PlexinC1 and all the plexins from mouse (Plexin A1, A2, A3, A4, B1, B2, B3, C1 and D1).

DOI: 10.7554/eLife.01279.011

Q1754E and K1775A mutations of mouse PlexinA3, equivalent to zebrafish PlexinC1 Q1032E and K1053A respectively, greatly impaired the cell collapse activity (Figure 9C). Mutations of Pro2032 in PlexinB1 and Lys1809 in PlexinB3, equivalent to Pro1050 and Lys1053 in zebrafish PlexinC1 respectively, have also been found in cancer patients (Cancer Genome Atlas Research Network, 2012; Seshagiri et al., 2012).

\section{Plexin induces the 'Gln63-in' conformation of Rap for catalysis}

Switch II of Rap in the complex structure adopts an unprecedented conformation that is markedly different from both the p120GAP/Ras and the RapGAP/Rap complexes (Figure 10) (Scheffzek et al., 1997; Scrima et al., 2008). Residues 60-63 in Switch II form a tight hairpin-like turn, which brings Gln63 close to $\mathrm{AlF}_{3}$ (therefore named the Gln63-in conformation). The Gln63 sidechain is placed in a nearly identical position in the active site as Gln61 in the p120GAP/Ras complex (Figure 10B). 
A
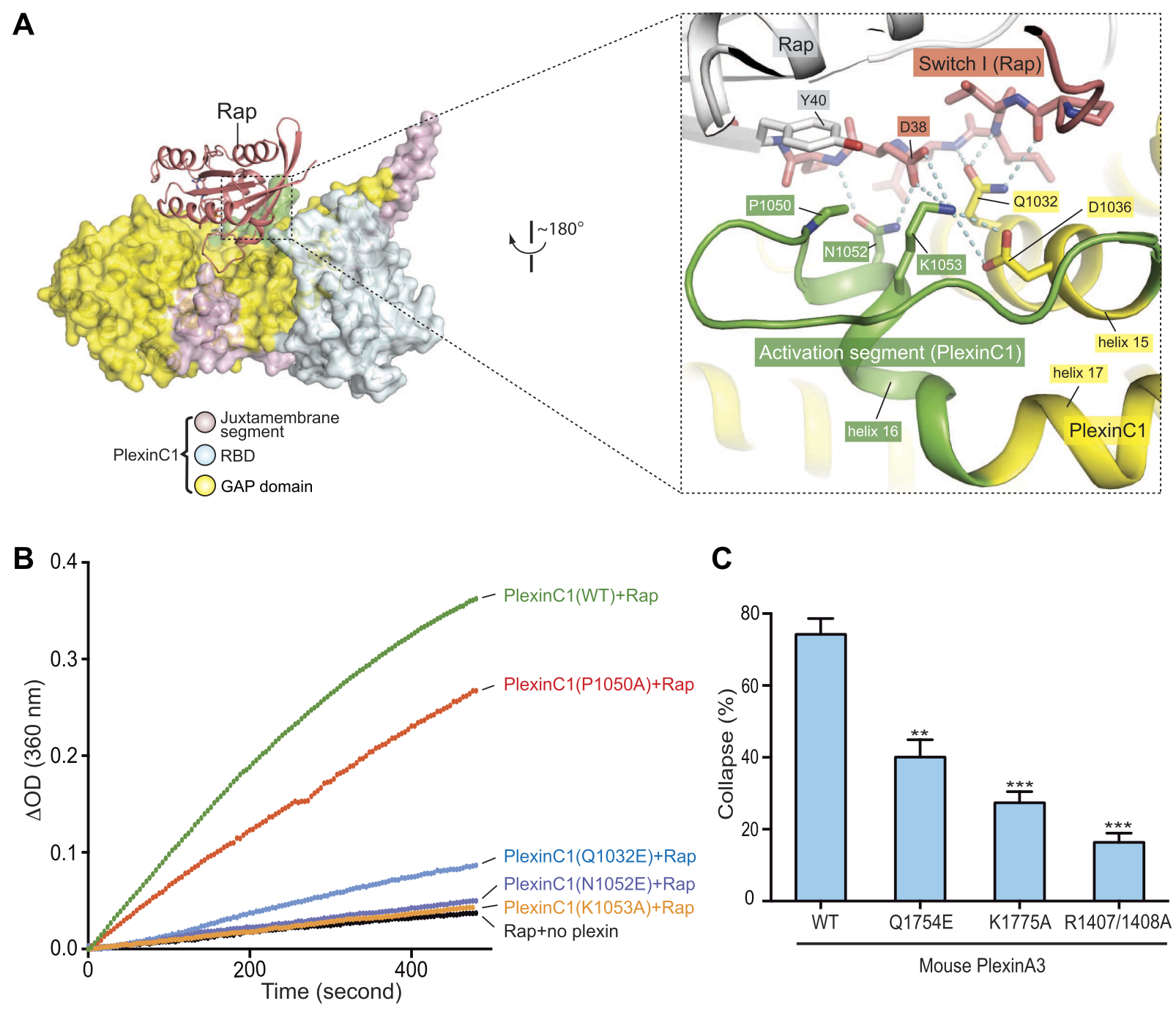

C

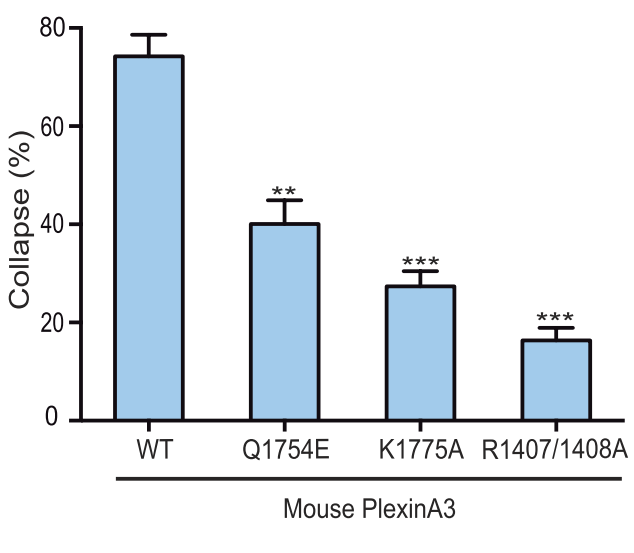

Figure 9. Interaction between the activation segment in PlexinC1 and Switch I in Rap. (A) Interface between the activation segment and Switch I. Polar interactions and potential hydrogen bonds are indicated by dashed lines. (B) GAP activity assays for mutations at the activation segment/Switch I interface. Monomeric PlexinC $1_{\text {cyto }}$ was used in these assays. The plots are representatives of three replicates. (C) COS7 cell collapse assays for mutations at the activation segment/Switch I interface. Q1754E, and K1775A of mouse PlexinA3 correspond to Q1032E and K1053A of zebrafish PlexinC1, respectively. The data analysis and presentation are the same as in Figure 7D.

DOI: $10.7554 /$ eLife.01279.012

This comparison strongly supports that Rap Gln63 indeed fulfills the catalytic role of Gln61 in Ras, that is stabilizing the nucleophilic water (Sot et al., 2010; Wang et al., 2012). Consistently, mutation of Gln63 in Rap has been shown to abolish GTP hydrolysis catalyzed by both plexin and the dualspecificity GAPs (Sot et al., 2010; Wang et alo, 2012). The segment following Gln63 (residues 64-67) adopts an extended conformation, allowing it to span the distance between Gln63 in the active site and the helix following Switch II. In contrast, the corresponding segments in the p120GAP/Ras and the Rap/RapGAP complexes adopt helical structures, holding residue 63 away from the active site (Figure 10B,C).

The Gln63-in conformation of Switch II is stabilized by numerous specific interactions between PlexinC1 and Rap. The side chains of Arg1001, Asn1005 and Asn1009 in helices 13 and 14 of PlexinC1 form a network of hydrogen bonds with the backbone of Switch II (Figure 10D). Pro611 in the second helix of the juxtamembrane segment makes van der Waals interactions with Thr65 in Switch II (Figure 10E). Mutation of either Asn1005 or Asn1009 dramatically decreased the GAP activity (Figure 10F). 
A
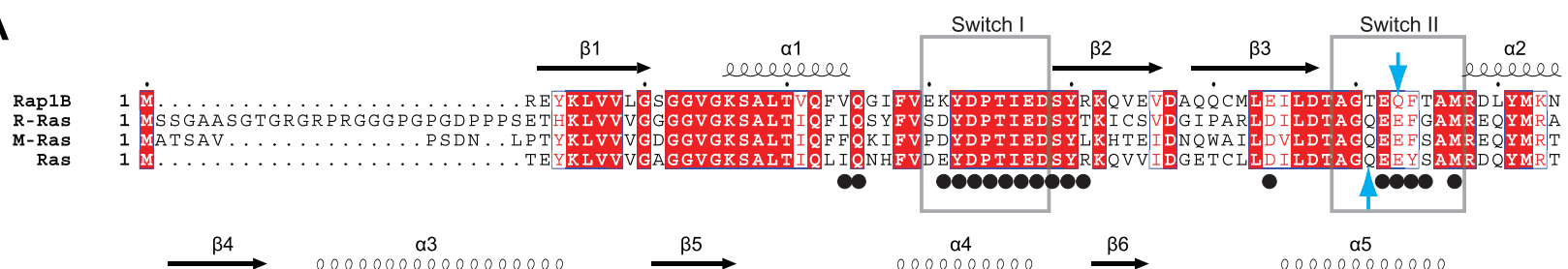

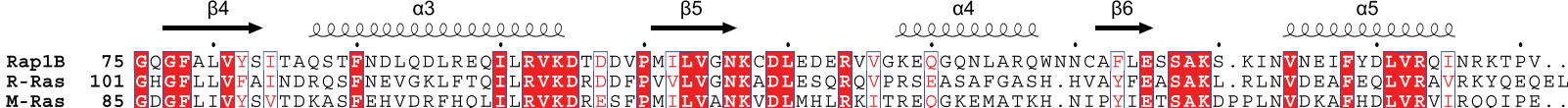

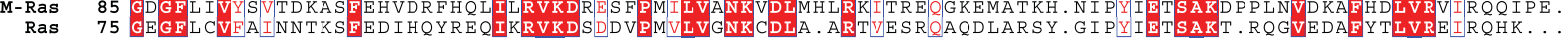

B

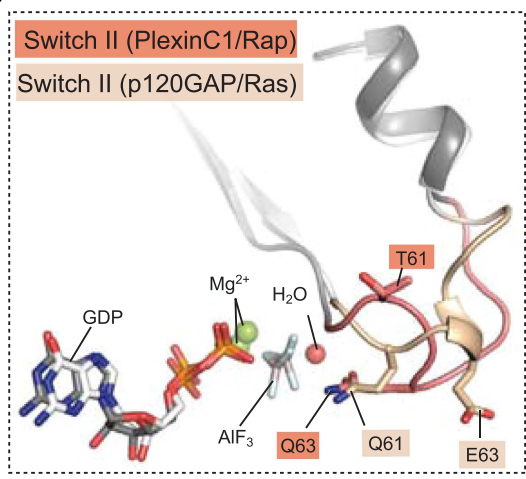

C

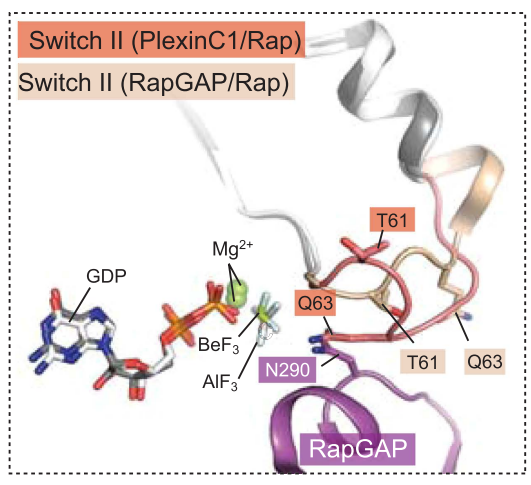

F

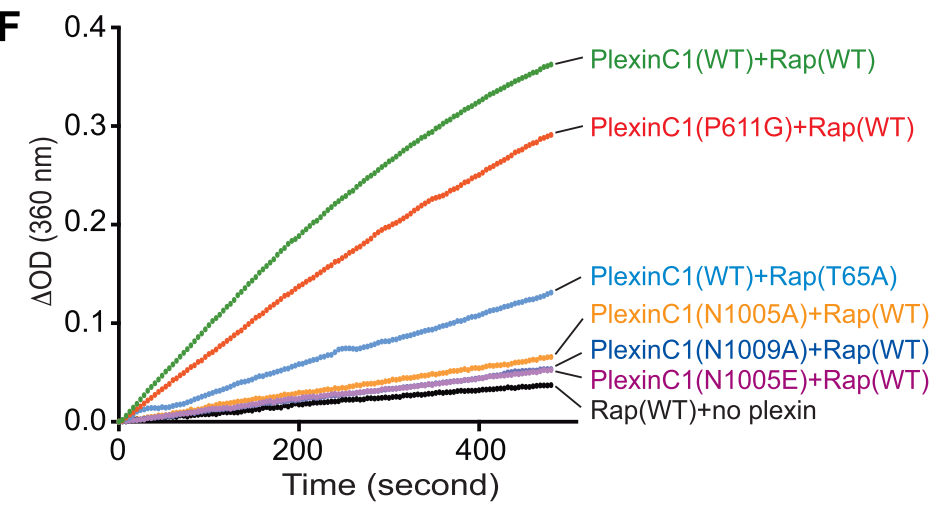

D

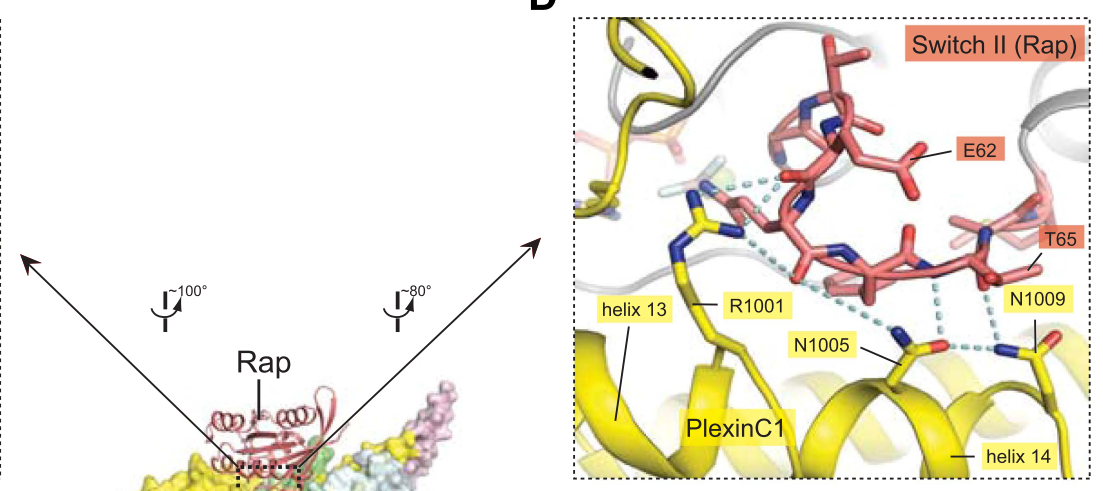

E

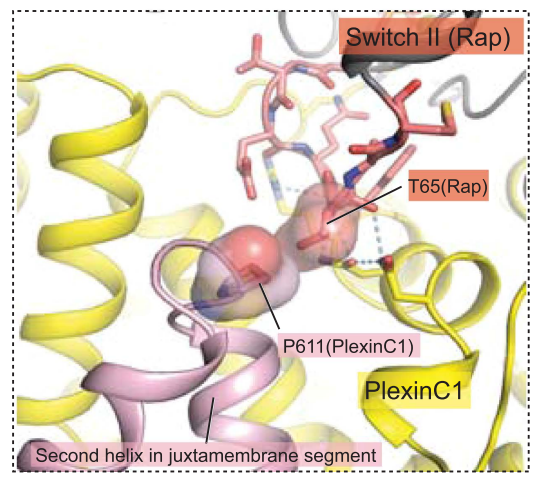

G

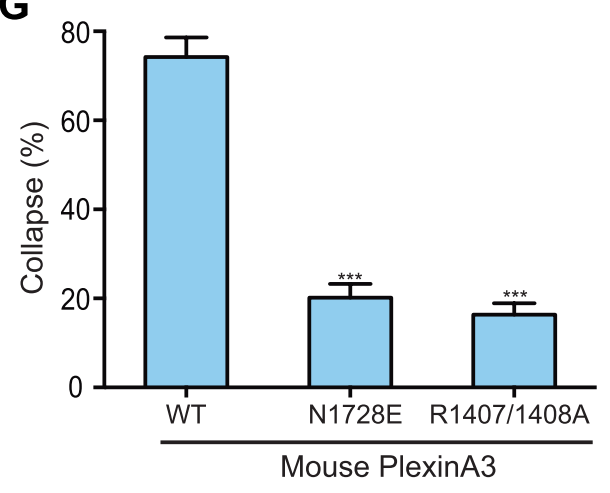

Figure 10. The Gln63-in conformation of Switch II in the PlexinC1 cyto/Rap1B complex. (A) Sequence alignment of human Rap1B, R-Ras, M-Ras and Ras. Black circles denote residues in Rap1B that are involved in binding PlexinC1 $1_{\text {cyto. }}$ Gln63 in Rap1B and Gln61 in Ras are highlighted by blue arrows. (B) Comparison of Switch II in the PlexinC1/Rap and the p120GAP/Ras (PDB ID: 1WQ1) complexes. The nucleophilic $\mathrm{H}_{2} \mathrm{O}$ is not included in the PlexinC1 $1_{\text {cyto }}$ Rap1B structure due to low resolution of the density map. (C) Comparison of Switch II in the PlexinC1/Rap and the RapGAP/Rap (PDB ID: 3BRW) complexes. Figure 10. Continued on next page 
Figure 10. Continued

(D) Specific interactions between PlexinC1 and Switch II in Rap1B. Polar interactions and potential hydrogen bonds are indicated by dashed lines. (E) Interaction between Pro611 in PlexinC1 and Thr65 in Rap1B. (F) GAP activity assays for mutations at the plexin/Switch II interface. Monomeric PlexinC $1_{\text {cyto }}$ was used in these assays. The plots are representatives of three replicates. (G) COS7 cell collapse assays for mutations at the plexin/Switch II interface. The data analysis and presentation are the same as in Figure 7D.

DOI: 10.7554/eLife.01279.013

The N1728E mutation in mouse PlexinA3 (equivalent to N1005E of zebrafish PlexinC1) also abolished the cell collapse activity (Figure 10G). Mutating Pro611 to glycine, which eliminates its interaction with Thr65 in Switch II, decreased the GAP activity (Figure 10F). Conversely, the wild-type PlexinC1 showed decreased activity towards the Rap T65A mutant (Figure 10F). The Switch II-interacting residues are highly conserved among the plexin family members, suggesting they all use the same mechanism to stabilize the Gln63-in conformation.

\section{Specificity determinants in plexin and the dual-specificity GAPs}

The dual-specificity GAPs do not share some of the Switch II-interacting residues with plexin (Figure 11). For example, Asn1005 in PlexinC1 is replaced by a proline in the dual-specificity GAPs (Pro585 in SynGAP) (Pena et alı, 2008), lacking the ability to stabilize the Gln63-in conformation of Rap through hydrogen bonds. This loss may be compensated by the extra domains outside of the GAP domain in the dual-specificity GAPs, which have been shown to be required for their RapGAP activity but not for the RasGAP activity (Kupzig et al., 2006; Pena et al., 2008; Kupzig et al., 2009; Sot et al., 2010). It has been suggested that the extra domains contribute to the catalysis by stabilizing a certain conformation of Switch II (Kupzig et al., 2006; Kupzig et al., 2009).

RasGAPs such as p120GAP and neurofibromin also contain a proline at the position of Asn1005 in PlexinC1. GAP1m, the only GAP1 family member that is active toward Ras but not Rap, has a valine at this position (Figure 11B). Proline-to-valine mutants of the dual-specificity GAP1 family members (Rasal, CAPRI and GAP1 IP4BP) remain active toward Ras, but lose activity toward Rap (Kupzig et al., 2009). The superimposition of the $120 \mathrm{GAP} /$ Ras and PlexinC1/Rap structures suggests the basis for how this residue determines the substrate specificity of these GAPs (Figure 11A). Pro907 in p120GAP contributes to Ras binding by stacking against Tyr64 in Switch II of Ras. A valine residue at the position of Pro907 (Val515 in GAP1m in Figure 11B) appears to be readily accommodated in this Ras binding mode (Figure 11A). Assuming Rap adopts the same Gln63-in conformation when it binds the dualspecificity GAPs, a proline residue at this position in the GAPs is compatible with the interaction. However, the Gln63-in conformation of Rap places Phe64 much closer to the proline residue (Figure 11A). Replacing the proline with a bulkier valine residue likely cause steric clashes with Phe64 in Rap, leading to loss of the RapGAP activity.

\section{Unique interactions between plexin and Rap sharpen the specificity}

In addition to Switch II, the PlexinC1/Rap interface involves several other residues in Rap that diverge from Ras/R-Ras/M-Ras. Residue 31 in Rap and Ras is a key residue for determining the binding specificity for downstream effectors of these two closely related small GTPases (Nassar et al., 1996). Our PlexinC $1_{\text {cyto }} /$ Rap1B structure suggests that residue 31 is also a determinant for the specificity between the plexin GAP and Rap. Rap possesses a lysine at this position, which is replaced by a negatively charged residue (aspartate or glutamate) in Ras/R-Ras/M-Ras (Figure 10A). Lys31 and Asp33 in Rap form a charge-charge pair and are buried by the activation segment in PlexinC1 (Figure 12B). We made a Rap(K31E) mutant to render it more similar to Ras/R-Ras/M-Ras. This mutation is predicted to destabilize the PlexinC1/Rap interaction, since it closely places two buried negative charges. The GAP assay indeed showed that PlexinC1 failed to catalyze GTP hydrolysis for the K31E mutant (Figure 12C).

A potential salt-bridge between Asp95 in Rap1B and Lys666 in PlexinC1 may also contribute to their interaction and specificity (Figure 12A). Consistent with this notion, Rap2 has a proline residue at position 95 and is less responsive to the plexin GAP (Wang et al., 2012). The corresponding residues in Ras, R-Ras and M-Ras are glutamine, lysine and arginine respectively (Figure 10A). Mutating Rap Asp95 to lysine, as in R-Ras, substantially decreased the rate of PlexinC1-catalyzed GTP hydrolysis (Figure 12C). Likewise, PlexinC1(K666D) displayed lower GAP activity than the wild-type PlexinC1 (Figure 12C). The PlexinC1(K666D) and Rap(D95K) charge-swapped pair only slightly restored the GTP hydrolysis activity (Figure 12C), which may be due to disruption of the electrostatic complementarity 


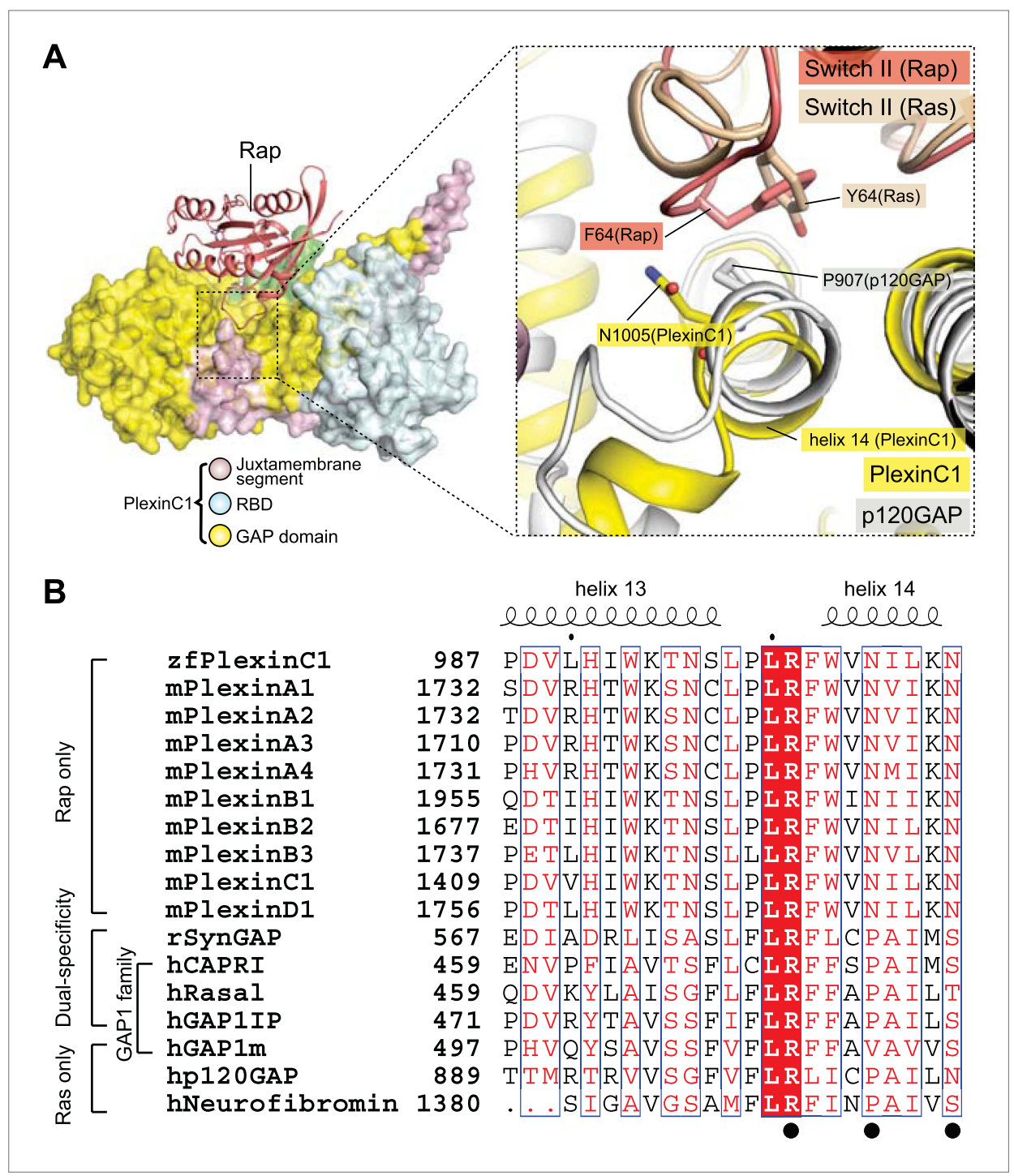

Figure 11. Comparison of the Switch Il-interacting region between plexin, RasGAPs and dual-specific GAPs. (A) Packing interactions made by Phe64 in Rap1B with PlexinC1 and Tyr64 in Ras with p120GAP. The PlexinC1 ctyol $_{\text {l }}$ Rap1B and the p120GAP/Ras structures are superimposed by using Rap1B and Ras as references. (B) Sequence alignment of the major Switch II-interacting segment in plexins, RasGAPs and dual-specificity GAPs. The black circles highlight the three residues (Arg1001, Asn1005 and Asn1009) in zebrafish PlexinC1 that make critical interactions with Switch II of Rap. zf: zebrafish; m: mouse; h: human; r: rat. DOI: 10.7554/eLife.01279.014

at the plexin/Rap interface by the mutations. We also tested the importance of this interaction in the cell-based assay, which showed that the equivalent mutation of mouse PlexinA3 (R1360D) impaired the cell collapse activity (Figure 12D). The same residue in human PlexinA1 (Arg1384) has been found mutated to cysteine in cancer patients (Seshagiri et al., 2012). These analyses together with the unique plexin/Switch II interface support the notion that plexins have evolved to recognize residues in Rap that have diverged from other Ras family members, leading to loss of activity toward Ras/R-Ras/M-Ras.

\section{Concluding remarks}

This study together with the previous structures of the plexin extracellular regions establishes a framework for understanding plexin regulation (Figure 13; Video 1) (Janssen et al., 2010; Liu et al., 2010; Nogi et al., 2010; Janssen et al., 2012). Semaphorin binding to the plexin extracellular region induces formation of the active dimer of the cytoplasmic region, which triggers its GAP activity to inactivate Rap through the non-canonical catalytic mechanism for signal transduction. Conformational 


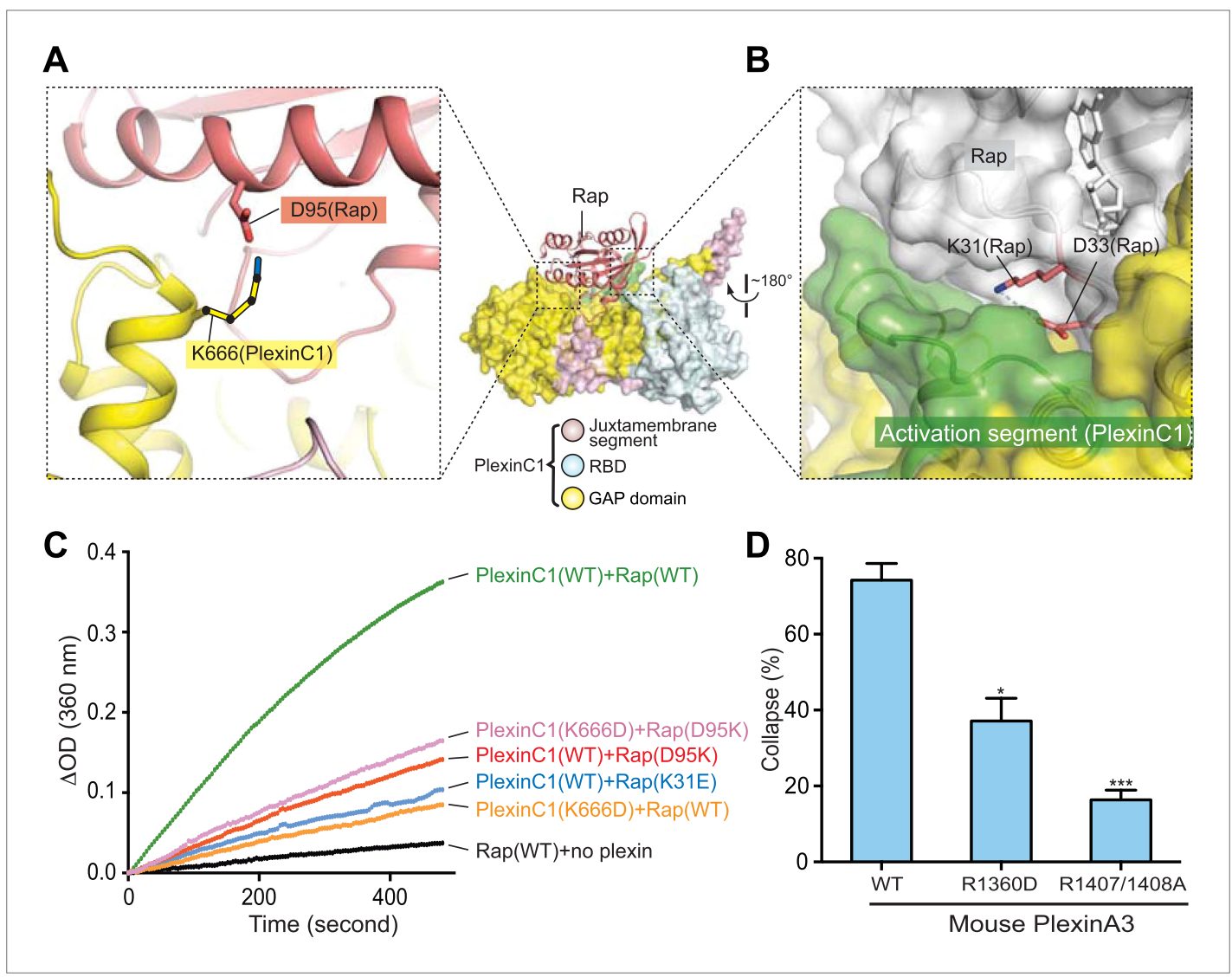

Figure 12. Additional specificity determinants in the PlexinC1/Rap1B complex. (A) Potential interaction between Lys666 in PlexinC1 and Asp95 in Rap. The side chain of Lys666 in PlexinC1 is not built in the final model due to weak electron density. It is modeled to show its potential interaction with Asp95 in Rap. (B) Burial of Lys31 in Rap1B at the PlexinC1/ Rap1B interface. (C) GAP activity assays for the specificity determinants. Monomeric PlexinC1 $1_{\text {cyto }}$ was used in these assays. The plots are representatives of three replicates. (D) COS7 cell collapse assays for the R1360D mutant of mouse PlexinA3 (equivalent to K666D of zebrafish PlexinC1). The data analysis and presentation are the same as in Figure 7D. DOI: 10.7554/eLife.01279.015

changes similar to those undertaken by the plexin GAP domain upon the dimerization may serve as on/off switches for other related GAPs such as CAPRI, which is also activated by dimerization (Dai et al., 2011). In addition to activation of the GAP, the dimerization-induced structural rearrangements may underlie the activation state-selective binding of plexins by signal transducers such as FARP2 (FERM, RhoGEF and pleckstrin homology protein 2) and MICAL (molecule interacting with CasL) (Toyofuku et al., 2005; Schmidt et al., 2008). The structures of the several extracellular membrane-proximal domains and the transmembrane helix of plexins have not been determined. Our data suggest that, upon semaphorin-induced dimerization, these domains are arranged precisely to ensure the proper juxtaposition of the juxtamembrane helix for inducing the active dimer of the cytoplasmic domain (Figure 13). Future work on these domains in the active dimeric state will fill in the missing links, leading to a complete structural model of semaphorin-activated plexin.

Clarifying the substrate specificity for the plexin GAP is essential for understanding plexin signaling. The results shown here and in our previous study (Wang et al., 2012) together demonstrate that while sharing the same domain fold with RasGAPs and dual-specificity GAPs, plexins are a unique group that are active to Rap, but not to Ras/R-Ras/M-Ras. Our analysis of the plexin/Rap complex structure reveals residues in both plexin and Rap that contribute to this specificity. P120GAP has been shown to bind GTP-bound Rap strongly but fail to catalyze its GTP hydrolysis, making Rap an effective inhibitor of the GAP activity of p120GAP to Ras/R-Ras/M-Ras (Frech et al., 1990; Hata et al., 1990; Yatani et al., 1991). The apparent GAP activity of plexins towards R-Ras and M-Ras reported previously may be caused indirectly by inactivation of Rap and alleviation of its inhibition on p120GAP. The induced GIn63-in 


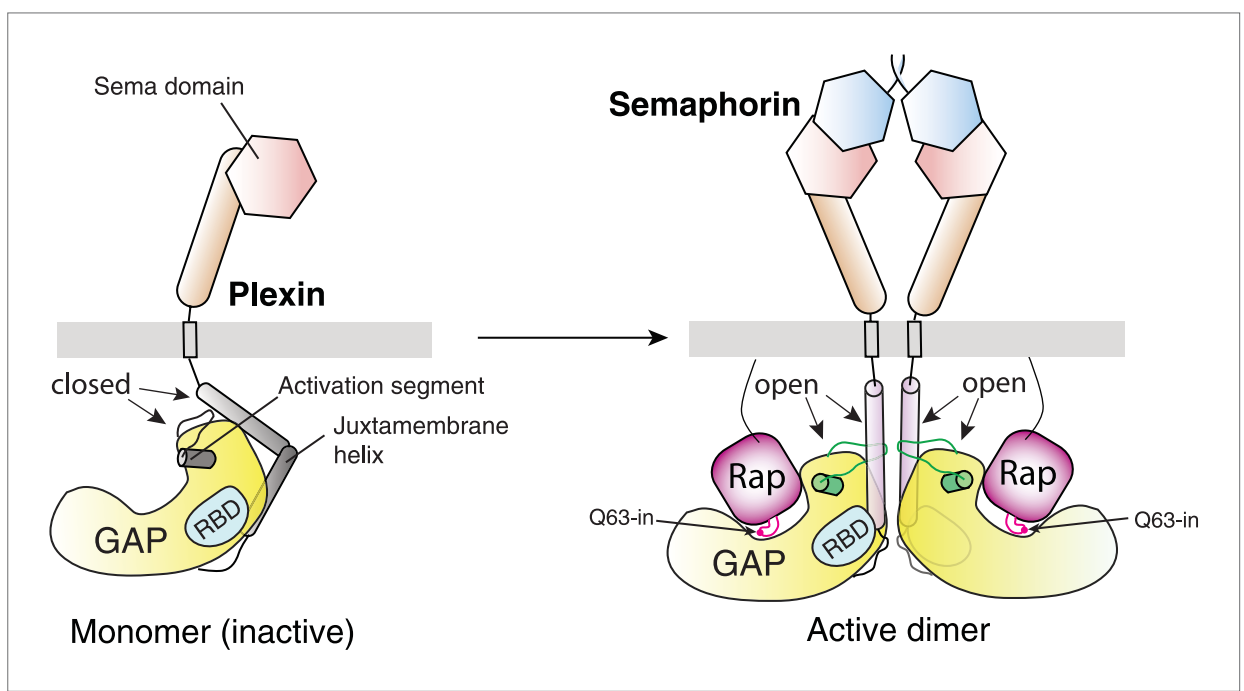

Figure 13. Schematic model for the activation of the plexin RapGAP by semaphorin-induced dimerization. DOI: 10.7554/eLife.01279.016

conformation of Rap seen in the PlexinC1/Rap complex structure likely represents the general mechanism by which plexins and the dual-specificity GAPs facilitate GTP hydrolysis for Rap. This conformation is stabilized by specific interactions made by several conserved residues in plexin. The dual-specificity GAPs achieve this through different mechanisms that likely involve the extra domains, the precise basis for which awaits structural studies of these GAPs in complex with Rap.

\section{Materials and methods}

\section{Protein expression}

The human Rap1B construct (residues 2-167) in a modified pET28 vector (Novagen, Darmstadt, Germany) that encodes a N-terminal $\mathrm{His}_{6}$-tag and a recognition site for the human rhinovirus $\mathrm{C} 3$

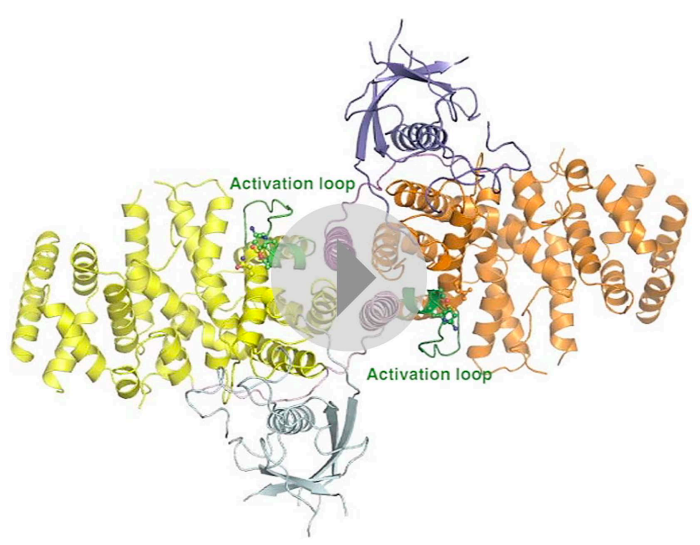

Video 1. Dimerization-induced activation of plexin cyto $_{\text {. }}$ and binding of Rap to the GAP active site. The video is based on the crystal structures of inactive monomeric PlexinA3 $3_{\text {cyto }}$ (PDB ID: $\left.3 \mid G 3\right), C C(a) P l e x i n C 1_{\text {cyto }}$ and the PlexinC $1_{\text {cyto }} /$ Rap1B complex. It is rendered for illustrating the dimerization-induced structural rearrangements and the binding mode between plexin and Rap. The actual order of the events and conformational transition trajectories likely do not follow those in the video. DOI: 10.7554/eLife.01279.017 protease has been described previously (Wang et al., 2012). The Rap1B constructs (2-166) containing a C-terminal flexible linker followed by a sortase recognition motif (one letter-code sequence: LPETGG) were generated by PCR and subcloned into the same vector. Seven versions of the linker were generated: 0-residue (containing the LPETGG motif only), 11-residue (sequence: GGSGGSGSGSS), 14-residue (sequence: SGGSGSGSSGGSGS), 16-residue (sequence: GGSGGSGSGSSGGSGS), 21 -residue (sequence: $G G S G G S G S G S S G G S G S$ GGGSG), 24-residue (sequence: SGGSGSGSSGG SGSGGGSGSGSSG) and 26-residue (sequence: GGSGGSGSGSSGGSGSGGGSGSGSSG). The vector encodes a glycine residue at the second position from the $\mathrm{N}$-terminus, which becomes the $\mathrm{N}$-terminal residue after removal of the methionine residue encoded by the start codon during protein expression. An N-terminal glycine on the Rap1B protein would hinder the sortase-mediated ligation with Plexin (see below) (Popp et al., 2009). To avoid this problem, the vector was mutated to replace the glycine residue with an aspartate using a 
Quickchange reaction (Stratagene, La Jolla, CA). The Rap1B proteins were expressed in the bacteria strain BL21 (DE3) and purified as described previously (Wang et al., 2012).

The coding region for the zebrafish PlexinC $1_{\text {cyto }}$ (residues 552-1153) with a $\mathrm{N}$-terminal di-glycine tag was synthesized (GenScript, Piscataway, NJ) based on the gene bank entry XM_685667.4. The region encoding residues $552-1147$ was subcloned into another modified $\mathrm{pET} 28$ vector containing a $\mathrm{N}$-terminal tandem $\mathrm{His}_{6}-\mathrm{SUMO}$ tag (Wang et al., 2012). The GCN4 coiled-coil motif was fused to the $\mathrm{N}$-terminus of the PlexinC1 $1_{\text {cyto }}$ (residues 553-1153) without the di-glycine motif by PCR. The fusion was subcloned into the same modified pET28 vector. Quikchange (Stratagene) was used to alter the residues at the junction between the coiled-coil and PlexinC1. The coiled-coil fusion constructs of mouse PlexinA $1_{\text {cyto }}$ were cloned by using similar procedures. The protein was expressed in the bacteria strain ArcticExpress (Stratagene) and purified as described previously (He et al., 2009; Wang et al., 2012). The His ${ }_{6}$-SUMO-tag was removed by treatment with the SUMO-specific protease Ulp1. For the construct containing the di-glycine encoding sequence, the Ulp1 treatment yielded the PlexinC1 protein with a N-terminal GG-tag. All mutants of Rap and plexins were generated by Quickchange reactions (Stratagene), and expressed and purified as the respective wild-type proteins.

\section{Sortase-mediated ligation}

Ligation of the N-terminal $\mathrm{His}_{6} / \mathrm{C}$-terminal LPETGG-tagged Rap1B and the N-terminal GG-tagged PlexinC1 was catalyzed by the transpeptidase activity of sortase from Staphylococcus aureus (plasmid provide by Dr Hidde Ploegh) (Popp et al., 2009). Sortase with a N-terminal His 6 -tag was expressed and purified by using Ni-NTA chromatography. Sortase first cleaves the peptide bond between the threonine and first glycine within the LPETGG motif in Rap1B. In the second step, the GG-tagged PlexinC1 is added to the threonine to regenerate a native peptide bond between the two proteins. The reaction mix contained Rap1B, PlexinC1 and sortase at 450, 69 and $25 \mu \mathrm{M}$ respectively. Reactions were performed at room temperature for $3 \mathrm{hr}$ with simultaneous dialysis to remove the di-glycine byproduct. The dialysis buffer contained $20 \mathrm{mM}$ Tris $\mathrm{pH} 8,150 \mathrm{mM} \mathrm{NaCl}, 10 \%$ glycerol, $2 \mathrm{mM} \mathrm{MgCl}$, $2 \mathrm{mM}$ DTT, $10 \mathrm{mM} \mathrm{CaCl}_{2}$. The ligated PlexinC1/Rap1B complex was purified by Ni-NTA, ion exchange and gel filtration chromatographic steps. The $\mathrm{N}$-terminal $\mathrm{His}_{6}$-tag was removed by treatment with the human rhinovirus $\mathrm{C} 3$ protease.

\section{In vitro GAP assays}

The GAP assay was performed by coupling release of inorganic phosphate during GTP hydrolysis to the purine nucleoside phosphorylase-catalyzed conversion of 2-amino-6-mercapto-7-methylpurine ribonucleoside to ribose-1-phosphate, which can be monitored photometrically at the wavelength of $360 \mathrm{~nm}$ (Webb and Hunter, 1992). For analyzing various structure-based mutations of CC(a)PlexinC1 $1_{\text {cytor }}$ the single turnover GAP assay was used (Wang et al., 2012). The concentration of plexin in the assays shown in Figure $7 C$ was $0.25 \mu \mathrm{M}$. In the assays shown in Figure $7 A, B$, the concentration of plexin was $2 \mu \mathrm{M}$. The concentration of Rap1B(GTP) was $120 \mu \mathrm{M}$. In the assays for analyzing various mutants of the PlexinC $1_{\text {cyto }}$ monomer and Rap1B, the concentrations of PlexinC1 and Rap $1 \mathrm{~B}(\mathrm{GTP})$ were $5 \mu \mathrm{M}$ and $60 \mu \mathrm{M}$ respectively.

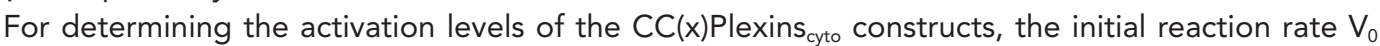
was measured at different Rap(GTP) concentrations ([S]) (Table 2). Fitting the data to the MichaelisMenten equation $\left(V_{0}=\left(V_{\max }[S]\right) /\left(K_{M}+[S]\right)\right)$ suggested that the Rap(GTP) concentrations used (25-150 $\left.\mu \mathrm{M}\right)$ were far below $K_{M}(>1 \mathrm{mM})$. For plexin constructs exhibiting low GAP activity, $V_{0}$ was determined by linear fitting of the initial period of the reaction (5-8 min) when less then $10 \%$ of Rap(GTP) had been hydrolyzed. After subtraction of the baseline rate from reaction without plexin, the $\mathrm{k}_{\text {cat }} / \mathrm{K}_{M}$ value of each construct was estimated by fitting the data to the equation $V_{0}=\left(K_{\text {cat }} / K_{M}\right)[E][S]$ (when [S] $<<K_{M}$ ), where $[E]$ is the total plexin concentration. For plexin constructs with high GAP activity, single turnover reaction curves measured at different Rap(GTP) concentrations were baseline-subtracted and simultaneously fitted to the single exponential equation: $A(t)=\left(A_{\max }-A_{\min }\right)(1-\exp (-k t))+A_{\min }$ where $k=\left(k_{\text {cat }} / K_{M}\right)[E]$. In the fitting, $k$ was treated as a global parameter. The plexin and Rap concentrations and the analysis methods used are listed in Table 2.

Due to the high intrinsic activity of the ligated plexin $_{\text {cyto }} /$ Rap complexes, all the bound GTP molecules were hydrolyzed to GDP during the purification process. To measure the GAP activity for these complexes, we used the multiple-turnover assay, in which $\left(\mathrm{NH}_{4}\right)_{2} \mathrm{SO}_{4}$ at $10 \mathrm{mM}$ and EDTA at $1 \mathrm{mM}$ are added to promote constant exchange of GTP/GDP for Rap in the complex, allowing continuous GTP hydrolysis provided sufficient GTP is present in the assay solution (Webb and Hunter, 1992). 
Table 2. Protein concentrations and fitting methods used for determining $k_{\mathrm{cat}} / \mathrm{K}_{M}$ of plexins

\begin{tabular}{|c|c|c|c|}
\hline Plexin construct & $\begin{array}{l}\text { Plexin concentration* } \\
(\mu \mathrm{M})\end{array}$ & $\begin{array}{l}\text { Rap-GTP concentrations } \\
(\mu \mathrm{M})\end{array}$ & Data fitting method $\dagger$ \\
\hline CC(a)PlexinA $1_{\text {cyto }}$ & 1.0 & $50.0,75.0,100.0,150.0$ & Single-exponential \\
\hline CC(b)Plexin $A 1_{\text {cyto }}$ & 5.0 & $50.0,75.0,100.0,150.0$ & Linear \\
\hline CC(c)PlexinA $1_{\text {cyto }}$ & 5.0 & $50.0,75.0,100.0,150.0$ & Linear \\
\hline CC(d)PlexinA $1_{\text {cyto }}$ & 1.0 & $50.0,75.0,100.0,150.0$ & Single-exponential \\
\hline CC(e)PlexinA $1_{\text {cyto }}$ & 5.0 & $50.0,75.0,100.0,150.0$ & Single-exponential \\
\hline$C C(f) P l e x i n A 1_{\text {cyto }}$ & 5.0 & $50.0,75.0,100.0,150.0$ & Linear \\
\hline$C C(g)$ Plexin $A 1_{\text {cyto }}$ & 1.0 & $50.0,75.0,100.0,150.0$ & Single-exponential \\
\hline Monomer PlexinC $1_{\text {cyto }}$ & 2.0 & $25.0,50.0,75.0,100.0,150.0$ & Linear \\
\hline $\mathrm{CC}(\mathrm{a})$ PlexinC1 $1_{\text {cyto }}$ & 2.0 & $50.0,75.0,100.0,150.0$ & Single-exponential \\
\hline$C C(b)$ PlexinC $1_{\text {cyto }}$ & 2.0 & $25.0,50.0,75.0,100.0,150.0$ & Linear \\
\hline$C C(d)$ Plexin $C 1_{\text {cyto }}$ & 2.0 & $50.0,75.0,100.0,150.0$ & Single-exponential \\
\hline CC(g)PlexinC $1_{\text {cyto }}$ & 2.0 & $25.0,50.0,75.0,100.0,150.0$ & Linear \\
\hline
\end{tabular}

*The plexin concentrations were chosen in order for the reaction rates to be within the dynamic range of the assay. TLinear fitting: $k_{\text {cat }} / K_{M}$ determined by fitting data to $V_{0}=\left(k_{\text {cat }} / K_{M}\right)[E][S] ;$ Single-exponential fitting: $k_{\text {cat }} / K_{M}$ determined by fitting data to $A(t)=\left(A_{\max }-A_{\min }\right)(1-\exp (-k t))+A_{\min ,}$ in which $k=k_{c a t} / K_{M}[E]$ and was fitted as a global parameter. DOI: 10.7554/eLife.01279.018

As the ligated complex with the 24-residue linker and the LPETGG motif crystallized and was used for structure determination, the same construct was chosen for extensive activity analyses at various concentrations. Unligated PlexinC1 and Rap1B mixed at the same concentrations were subjected to the same assay for comparison.

\section{Analytical ultracentrifugation}

Sedimentation velocity analytical ultracentrifugation experiments were carried out using the ligated PlexinC1 $1_{\text {cyto }} /$ Rap1B complex with the 24-residue linker and the LPETGG motif. Protein samples were prepared in Centrifugation Buffer (10 $\mathrm{mM}$ Tris $\mathrm{pH} 8,50 \mathrm{mM} \mathrm{NaCl}, 2 \mathrm{mM}$ TCEP, and $2 \mathrm{mM} \mathrm{MgCl}$ ). Samples at 0.5, 4, and $20 \mu \mathrm{M}$ were used for the experiments without $\mathrm{AlF}_{\mathrm{x}}$. Samples at 0.5, 4, 8 and 20 $\mu \mathrm{M}$ were used for the experiments in the presence of $2 \mathrm{mM} \mathrm{AlF}_{\mathrm{x}}$. All samples were equilibrated $\sim 14 \mathrm{hr}$ at $4^{\circ} \mathrm{C}$, then $\sim 400 \mu \mathrm{l}$ of the samples were loaded into the 'sample' sides of dual-sectored charcoal-filled Epon centerpieces that were sandwiched between sapphire windows in a cell housing; the 'reference' sectors were filled with the same volume of Centrifugation Buffer. Filled cells were placed in an An50Ti rotor and equilibrated for $2.5 \mathrm{hr}$ under vacuum in the centrifuge at $20^{\circ} \mathrm{C}$ prior to centrifugation. Experiments were conducted using a Beckman Optima XL-I analytical ultracentrifuge at 42,000 rpm at $20^{\circ} \mathrm{C}$. Absorbance data at $280 \mathrm{~nm}$ were collected using the Beckman control software until all components had fully sedimented. Protein partial-specific volume, solvent viscosity, and density values were calculated using the program Sednterp (Laue et al., 1992). The data were analyzed using the $c(s)$ distribution in the program SEDFIT (Schuck, 2000). A regularization level of 0.68 was routinely employed. Time-invariant noise elements were removed from the data (Schuck and Demeler, 1999). Data-acquisition timestamp errors (Zhao et al., 2013) were examined with SEDFIT and were found to be $\sim 0.1 \%$; we deemed this small error acceptable and did not correct the timestamps. Plots were generated with the program GUSSI (http://biophysics.swmed.edu/MBR/software.html).

\section{Crystallization and structure determination}

Mouse CC(d)PlexinA1, CC(g)PlexinA1, zebrafish CC(d)PlexinC1 and CC(a)PlexinC1 were subjected to crystallization trials. CC(a)PlexinC $1_{\text {cyto }}$ at $8 \mathrm{mg} / \mathrm{ml}$ crystallized initially at $20^{\circ} \mathrm{C}$ in $0.1 \mathrm{M}$ Bicine, $\mathrm{pH} 9.0$, $20 \%$ PEG 6000 in sitting-drop 96-well plates. Larger crystals were grown by sitting-drop vapor diffusion at $20^{\circ} \mathrm{C}$ in $0.1 \mathrm{M}$ Bis-Tris propane, $\mathrm{pH} 9.1,21 \%$ PEG 6000 . Crystals were cryo-protected using the crystallization solution supplemented with $25 \%$ glycerol and flash cooled in liquid nitrogen. Diffraction data were collected at $100 \mathrm{~K}$ on beamline 19ID at the Advanced Photon Source (Argonne National 
Laboratory). Data were indexed, integrated and scaled by using HKL2000 (Otwinowski and Minor, 1997). A $3.3 \AA$ dataset in the $\mathrm{P} 2{ }_{1} 2{ }_{1} 2_{1}$ space group was collected. The 'autocorrections' option in HKL2000 was selected to truncate and scale the anisotropic data, which was then converted to the mtz format by using the Ctruncate program in CCP4 (Padilla and Yeates, 2003; Winn et al., 2011). The structure of the GAP domain of mouse PlexinA3 (PDB ID: 3IG3) was used as the molecular replacement search model using the Phaser module in the Phenix package (Adams et al., 2002; Mccoy et al., 2007).

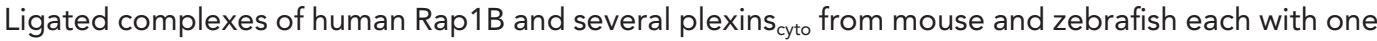
of the 7 versions of the linker mentioned above were all subjected to crystallization trails. The ligated complex of zebrafish PlexinC $1_{\text {cyto }}$ and human Rap1B with the 24-residue linker and the LPETGG-tag at $4 \mathrm{mg} / \mathrm{ml}$ crystallized initially at $20^{\circ} \mathrm{C}$ in $0.1 \mathrm{M} \mathrm{HEPES} \mathrm{pH} \mathrm{7.5,10 \%} \mathrm{2-propanol,} 20 \%$ PEG $4 \mathrm{~K}$ in sittingdrop 96-well plates. Larger crystals were grown by hanging-drop vapor diffusion at $20^{\circ} \mathrm{C}$ in $0.1 \mathrm{M}$ HEPES pH 7.3, 5\% 2-propanol, 25\% PEG 3350, 3.6\% polypropylene glycol P400. Cryo-protection of the crystals was achieved using with the crystallization solution supplemented with $25 \%$ glycerol. Cryoprotected crystals were snap cooled in liquid nitrogen. The data collection and processing were performed in a similar manner as described for the CC(a)PlexinC $1_{\text {cyto }}$ crystal, expect that the 'autocorrections' option was not used. The diffract pattern extended to $3.3 \AA$ and was consistent with the symmetry of the P1 space group. One protomer from the $C C(a) P l e x i n C 1_{\text {cyto }}$ structure was used as the molecular replacement search model for plexin. The structure of Rap1B from the Rap1B/RapGAP complex (PDB ID: 3BRW) was used as the search model for Rap1B.

Iterative model building and refinement were performed using the Phenix and Coot programs respectively (Adams et al., 2002; Emsley and Cowtan, 2004). In the PlexinC $1_{\text {cyto }} /$ Rap1B structure, the linker between the $\mathrm{C}$-terminus of Rap1B and the $\mathrm{N}$-terminus of PlexinC $1_{\text {cyto }}$ is not included in the final model due to lack of discernable electron density. Assuming the complexes in the crystal are formed by the covalently linked pairs of Rap1B and PlexinC $1_{\text {cyto, }}$ the linker and the disordered flanking residues from the two proteins (a total of $\sim 32$ residues) are sufficient for spanning the $\sim 45 \AA$ distance between the two ends without imposing restraints on the plexin/Rap binding mode (Figure 8A, middle panel). The structural superimpositions shown in the Figures 5 and 6 were based on helices 13,14 and 15 in the plexin GAP domain, because they are at the center of the GAP active site and adopt highly similar conformations in all the plexin structures. Comprehensive model validation was performed by using MolProbity (Chen et al., 2010). Detailed statistics of data collection and refinement are listed in Table 1. Structure figures were prepared in PyMOL (the PyMOL Molecular Graphics System, Schrodinger). Sequences were aligned by using T-Coffee (Notredame et al., 2000) and rendered with ESPript (Gouet et al., 1999). Molecular surface area was calculated using the get_area function in PyMOL. Morph frames in Video 1 were generated by using the Yale morph server (Krebs and Gerstein, 2000) and rendered in PyMOL.

\section{COS7 cell collapse assay}

Mutants of mouse PlexinA3 were designed based on a sequence alignment of zebrafish PlexinC1 with all mouse plexins (Plexin A1, A2, A3, A4, B1, B2, B3, C1 and D1). COS7 cell collapse assays using full-length mouse PlexinA3 were performed as described previously (He et al., 2009). Briefly, $1 \times 10^{5}$ COS7 cells were plated in each well of a 6-well plate one day prior to transfection. FuGENE 6 (Promega, Madison, WI) was used to transfect each well with PlexinA3 (1 $\mathrm{gg}$ plasmid) and the co-receptor Neuropilin2 $(0.5 \mu \mathrm{g}$ plasmid) following the manufacturer's instructions. 2 days post transfection, $5 \mathrm{nM}$ alkaline phosphatase-tagged Sema3F was added to each well and incubated for $25 \mathrm{~min}$ at $37^{\circ} \mathrm{C}$. The cells were washed, fixed and heat-treated at $65^{\circ} \mathrm{C}$ for $1 \mathrm{hr}$ to inactivate endogenous phosphatases. Cells were stained with the BCIP/NBT alkaline phosphatase substrate (Sigma, St. Louis, MO), and counted using a randomized and blind method.

\section{Acknowledgements}

We thank Andrew Schober, Josee Santiago and members in the Zhang laboratory for discussions and technical assistance. We also thank Dr Hidde Ploegh at the Whitehead Institute for providing the sortase construct, Dominika Borek, Zhe Chen, the staff at the structural biology laboratory at UTSW and at beamline 19ID of Advanced Photon Source for assistance with X-ray data collection and processing. $\mathrm{XZ}$ is a Virginia Murchison Linthicum Scholar in Medical Research at UTSW. Results shown in this report are derived from work performed at Argonne National Laboratory, Structural Biology Center at the 
Advanced Photon Source. Argonne is operated by U. Chicago Argonne, LLC, for the US Department of Energy, Office of Biological and Environmental Research under contract DE-AC02-06CH11357.

\section{Additional information}

Funding

\begin{tabular}{lll} 
Funder & Grant reference number & Author \\
\hline National Institutes of Health & GM088197 & $\begin{array}{l}\text { Yuxiao Wang, } \\
\text { Heath G Pascoe, } \\
\end{array}$ \\
& & $\begin{array}{l}\text { Huawei He, } \\
\text { Xuewu Zhang }\end{array}$ \\
\hline The Welch foundation & I-1702 & $\begin{array}{l}\text { Yuxiao Wang, } \\
\text { Huawei He, } \\
\text { Xuewu Zhang }\end{array}$ \\
\hline National Institutes of Health & GM008203 & Heath G Pascoe
\end{tabular}

The funders had no role in study design, data collection and interpretation, or the decision to submit the work for publication.

Author contributions

YW, HGP, XZ, Conception and design, Acquisition of data, Analysis and interpretation of data, Drafting or revising the article; $C A B$, Acquisition of data, Analysis and interpretation of data, Drafting or revising the article; $\mathrm{HH}$, Conception and design, Acquisition of data, Drafting or revising the article

\section{Additional files}

Major dataset

The following datasets were generated:

\begin{tabular}{|c|c|c|c|c|}
\hline Author(s) & Year & Dataset title & Dataset ID and/or URL & $\begin{array}{l}\text { Database, license, } \\
\text { and accessibility } \\
\text { information }\end{array}$ \\
\hline $\begin{array}{l}\text { Pascoe HG, } \\
\text { Zhang } X\end{array}$ & 2013 & $\begin{array}{l}\text { Crystal structure of } \\
\text { the PlexinC1/Rap1B } \\
\text { complex }\end{array}$ & $\begin{array}{l}\text { http://www.rcsb.org/pdb/ } \\
\text { explore/explore. } \\
\text { do?structureld=4M8N }\end{array}$ & $\begin{array}{l}\text { Publicly available at the } \\
\text { Protein Data Bank (http:// } \\
\text { www.rcsb.org/pdb/). }\end{array}$ \\
\hline $\begin{array}{l}\text { Wang } Y, \\
\text { Zhang } X\end{array}$ & 2013 & $\begin{array}{l}\text { Crystal structure of the active } \\
\text { dimer of zebrafish PlexinC1 } \\
\text { cytoplasmic region }\end{array}$ & $\begin{array}{l}\text { http://www.rcsb.org/pdb/ } \\
\text { explore/explore. } \\
\text { do?structureld=4M8M }\end{array}$ & $\begin{array}{l}\text { Publicly available at the } \\
\text { Protein Data Bank (http:// } \\
\text { www.rcsb.org/pdb/). }\end{array}$ \\
\hline
\end{tabular}

\section{References}

Adams PD, Grosse-Kunstleve RW, Hung LW, loerger TR, McCoy AJ, Moriarty NW, et al. 2002. PHENIX: building new software for automated crystallographic structure determination. Acta Crystallogr 58:1948-54. doi: 10.1107/ S0907444902016657.

Antipenko A, Himanen JP, van Leyen K, Nardi-Dei V, Lesniak J, Barton WA, et al. 2003. Structure of the semaphorin3A receptor binding module. Neuron 39:589-98. doi: 10.1016/S0896-6273(03)00502-6.

Bell CH, Aricescu AR, Jones EY, Siebold C. 2011. A dual binding mode for RhoGTPases in plexin signalling. PLOS Biol 9:e1001134. doi: 10.1371/journal.pbio.1001134.

Bos JL, Rehmann H, Wittinghofer A. 2007. GEFs and GAPs: critical elements in the control of small G proteins. Cell 129:865-77. doi: 10.1016/j.cell.2007.05.018.

Cancer Genome Atlas Research Network. 2012. Comprehensive molecular characterization of human colon and rectal cancer. Nature 487:330-7. doi: 10.1038/nature11252.

Cancer Genome Atlas Research Network. 2012. Comprehensive genomic characterization of squamous cell lung cancers. Nature 489:519-25. doi: 10.1038/nature11404.

Chen VB, Arendall WB III, Headd JJ, Keedy DA, Immormino RM, Kapral GJ, et al. 2010. MolProbity: all-atom structure validation for macromolecular crystallography. Acta Crystallogr 66:12-21. doi: 10.1107/ S0907444909042073.

Dai Y, Walker SA, de Vet E, Cook S, Welch HC, Lockyer PJ. 2011. Ca2+-dependent monomer and dimer formation switches CAPRI protein between ras GTPase-activating protein (GAP) and RapGAP activities. J Biol Chem 286:19905-16. doi: 10.1074/jbc.M110.201301. 
Driessens MH, Hu H, Nobes CD, Self A, Jordens I, Goodman CS, et al. 2001. Plexin-B semaphorin receptors interact directly with active Rac and regulate the actin cytoskeleton by activating Rho. Curr Biol 11:339-44. doi: 10.1016/S0960-9822(01)00092-6.

Emsley P, Cowtan K. 2004. Coot: model-building tools for molecular graphics. Acta Crystallogr 60:2126-32. doi: 10.1107/S0907444904019158.

Frech M, John J, Pizon V, Chardin P, Tavitian A, Clark R, et al. 1990. Inhibition of GTPase activating protein stimulation of Ras-p21 GTPase by the Krev-1 gene product. Science 249:169-71. doi: 10.1126/science.2164710.

Gloerich M, Bos JL. 2011. Regulating Rap small G-proteins in time and space. Trends Cell Biol 21:615-23. doi: 10.1016/j.tcb.2011.07.001.

Gouet P, Courcelle E, Stuart DI, Metoz F. 1999. ESPript: analysis of multiple sequence alignments in PostScript. Bioinformatics 15:305-8.

Gu C, Giraudo E. 2013. The role of semaphorins and their receptors in vascular development and cancer. Exp Cell Res 319:1306-16. doi: 10.1016/j.yexcr.2013.02.003.

Gui Y, Guo G, Huang Y, Hu X, Tang A, Gao S, et al. 2011. Frequent mutations of chromatin remodeling genes in transitional cell carcinoma of the bladder. Nat Genet 43:875-8. doi: 10.1038/ng.907.

Hata Y, Kikuchi A, Sasaki T, Schaber MD, Gibbs JB, Takai Y. 1990. Inhibition of the ras p21 GTPase-activating protein-stimulated GTPase activity of c-Ha-ras p21 by smg p21 having the same putative effector domain as ras p21s. J Biol Chem 265:7104-7.

He H, Yang T, Terman JR, Zhang X. 2009. Crystal structure of the plexin A3 intracellular region reveals an autoinhibited conformation through active site sequestration. Proc Natl Acad Sci USA 106:15610-5. doi: 10.1073/pnas.0906923106.

Hu H, Marton TF, Goodman CS. 2001. Plexin B mediates axon guidance in Drosophila by simultaneously inhibiting active Rac and enhancing RhoA signaling. Neuron 32:39-51. doi: 10.1016/S0896-6273(01)00453-6.

Huse M, Kuriyan J. 2002. The conformational plasticity of protein kinases. Cell 109:275-82. doi: 10.1016/ S0092-8674(02)00741-9.

Janssen BJ, Malinauskas T, Weir GA, Cader MZ, Siebold C, Jones EY. 2012. Neuropilins lock secreted semaphorins onto plexins in a ternary signaling complex. Nat Struct Mol Biol 19:1293-9. doi: 10.1038/nsmb.2416.

Janssen BJ, Robinson RA, Perez-Branguli F, Bell CH, Mitchell KJ, Siebold C, et al. 2010. Structural basis of semaphorinplexin signalling. Nature 467:1118-22. doi: 10.1038/nature09468.

Klostermann A, Lohrum M, Adams RH, Puschel AW. 1998. The chemorepulsive activity of the axonal guidance signal semaphorin D requires dimerization. J Biol Chem 273:7326-31. doi: 10.1074/jbc.273.13.7326.

Koppel AM, Raper JA. 1998. Collapsin-1 covalently dimerizes, and dimerization is necessary for collapsing activity. J Biol Chem 273:15708-13. doi: 10.1074/jbc.273.25.15708.

Krebs WG, Gerstein M. 2000. The morph server: a standardized system for analyzing and visualizing macromolecular motions in a database framework. Nucleic Acids Res 28:1665-75. doi: 10.1093/nar/28.8.1665.

Kupzig S, Bouyoucef-Cherchalli D, Yarwood S, Sessions R, Cullen PJ. 2009. The ability of GAP1IP4BP to function as a Rap1 GTPase-activating protein (GAP) requires its Ras GAP-related domain and an arginine finger rather than an asparagine thumb. Mol Cell Biol 29:3929-40. doi: 10.1128/MCB.00427-09.

Kupzig S, Deaconescu D, Bouyoucef D, Walker SA, Liu Q, Polte CL, et al. 2006. GAP1 family members constitute bifunctional Ras and Rap GTPase-activating proteins. J Biol Chem 281:9891-900. doi: 10.1074/jbc. M512802200.

Laue TM, Shah BD, Ridgeway TM, Pelletier SL. 1992. Computer-aided interpretation of analytical sedimentation data for proteins. In: Harding SE, Rowe AJ, Horton JC, editors. Analytical ultracentrifugation in biochemistry and polymer science. Cambridge, United Kingdom: Royal Society of Chemistry. p. 90-125.

Li S, Nakamura S, Hattori S. 1997. Activation of R-Ras GTPase by GTPase-activating proteins for Ras, Gap1(m), and p120GAP. J Biol Chem 272:19328-32. doi: 10.1074/jbc.272.31.19328.

Liu H, Juo ZS, Shim AH, Focia PJ, Chen X, Garcia KC, et al. 2010. Structural basis of semaphorin-plexin recognition and viral mimicry from Sema7A and A39R complexes with PlexinC1. Cell 142:749-61. doi: 10.1016/j.cell.2010.07.040.

Love CA, Harlos K, Mavaddat N, Davis SJ, Stuart DI, Jones EY, et al. 2003. The ligand-binding face of the semaphorins revealed by the high-resolution crystal structure of SEMA4D. Nat Struct Biol 10:843-8. doi: 10.1038/nsb977.

Mccoy AJ, Grosse-Kunstleve RW, Adams PD, Winn MD, Storoni LC, Read RJ. 2007. Phaser crystallographic software. J App/ Crystallogr 40:658-74. doi: 10.1107/S0021889807021206.

Nassar N, Horn G, Herrmann C, Block C, Janknecht R, Wittinghofer A. 1996. Ras/Rap effector specificity determined by charge reversal. Nat Struct Biol 3:723-9. doi: 10.1038/nsb0896-723.

Nogi T, Yasui N, Mihara E, Matsunaga Y, Noda M, Yamashita N, et al. 2010. Structural basis for semaphorin signalling through the plexin receptor. Nature 467:1123-7. doi: 10.1038/nature09473.

Notredame C, Higgins DG, Heringa J. 2000. T-Coffee: a novel method for fast and accurate multiple sequence alignment. J Mol Biol 302:205-17. doi: 10.1006/jmbi.2000.4042.

Ohba Y, Mochizuki N, Yamashita S, Chan AM, Schrader JW, Hattori S, et al. 2000. Regulatory proteins of r-ras, TC21/R-Ras2, and m-Ras/R-Ras3. J Biol Chem 275:20020-6. doi: 10.1074/jbc.M000981200.

Oinuma I, Ishikawa Y, Katoh H, Negishi M. 2004. The Semaphorin 4D receptor Plexin-B1 is a GTPase activating protein for R-Ras. Science 305:862-5. doi: 10.1126/science.1097545.

O'Shea EK, Klemm JD, Kim PS, Alber T. 1991. X-ray structure of the GCN4 leucine zipper, a two-stranded, parallel coiled coil. Science 254:539-44. doi: 10.1126/science.1948029.

Otwinowski Z, Minor W. 1997. Processing of x-ray diffraction data collected in oscillation mode. Methods Enzymol 276:307-26. doi: 10.1016/S0076-6879(97)76066-X. 
Padilla JE, Yeates TO. 2003. A statistic for local intensity differences: robustness to anisotropy and pseudo-centering and utility for detecting twinning. Acta Crystallogr D Biol Crystallogr 59:1124-30. doi: 10.1107/S0907444903007947.

Pena V, Hothorn M, Eberth A, Kaschau N, Parret A, Gremer L, et al. 2008. The C2 domain of SynGAP is essential for stimulation of the Rap GTPase reaction. EMBO Rep 9:350-5. doi: 10.1038/embor.2008.20.

Perrot V, Vazquez-Prado J, Gutkind JS. 2002. Plexin B regulates Rho through the guanine nucleotide exchange factors leukemia-associated Rho GEF (LARG) and PDZ-RhoGEF. J Biol Chem 277:43115-20. doi: 10.1074/jbc.M206005200.

Popp MW, Antos JM, Ploegh HL. 2009. Site-specific protein labeling via sortase-mediated transpeptidation. Curr Protoc Protein Sci Chapter 15:Unit 15 13. doi: 10.1002/0471140864.ps1503s56.

Quilliam LA, Castro AF, Rogers-Graham KS, Martin CB, Der CJ, Bi C. 1999. M-Ras/R-Ras3, a transforming ras protein regulated by Sos1, GRF1, and p120 Ras GTPase-activating protein, interacts with the putative Ras effector AF6. J Biol Chem 274:23850-7. doi: 10.1074/jbc.274.34.23850.

Rohm B, Rahim B, Kleiber B, Hovatta I, Püschel AW. 2000. The semaphorin 3A receptor may directly regulate the activity of small GTPases. FEBS Lett 486:68-72. doi: 10.1016/S0014-5793(00)02240-7.

Saito Y, Oinuma I, Fujimoto S, Negishi M. 2009. Plexin-B1 is a GTPase activating protein for M-Ras, remodelling dendrite morphology. EMBO Rep 10:614-21. doi: 10.1038/embor.2009.63.

Sakurai A, Doci C, Gutkind JS. 2012. Semaphorin signaling in angiogenesis, lymphangiogenesis and cancer. Cell Res 22:23-32. doi: 10.1038/cr.2011.198.

Scheffzek K, Ahmadian MR, Kabsch W, Wiesmuller L, Lautwein A, et al. 1997. The Ras-RasGAP complex: structural basis for GTPase activation and its loss in oncogenic Ras mutants. Science 277:333-8. doi: 10.1126/science.277.5324.333.

Scheffzek K, Ahmadian MR, Wiesmuller L, Kabsch W, Stege P, Schmitz F, et al. 1998. Structural analysis of the GAP-related domain from neurofibromin and its implications. EMBO J 17:4313-27. doi: 10.1093/emboj/17.15.4313.

Schmidt EF, Shim SO, Strittmatter SM. 2008. Release of MICAL autoinhibition by semaphorin-plexin signaling promotes interaction with collapsin response mediator protein. J Neurosci 28:2287-97. doi: 10.1523/JNEUROSCI.5646-07.2008.

Schuck P. 2000. Size-distribution analysis of macromolecules by sedimentation velocity ultracentrifugation and lamm equation modeling. Biophys J 78:1606-19. doi: 10.1016/S0006-3495(00)76713-0.

Schuck P, Demeler B. 1999. Direct sedimentation analysis of interference optical data in analytical ultracentrifugation. Biophys J 76:2288-96. doi: 10.1016/S0006-3495(99)77384-4.

Scrima A, Thomas C, Deaconescu D, Wittinghofer A. 2008. The Rap-RapGAP complex: GTP hydrolysis without catalytic glutamine and arginine residues. EMBO J 27:1145-53. doi: 10.1038/emboj.2008.30.

Seshagiri S, Stawiski EW, Durinck S, Modrusan Z, Storm EE, Conboy CB, et al. 2012. Recurrent R-spondin fusions in colon cancer. Nature 488:660-4. doi: 10.1038/nature11282.

Siebold C, Jones EY. 2013. Structural insights into semaphorins and their receptors. Semin Cell Dev Biol 24:139-45. doi: 10.1016/j.semcdb.2012.11.003.

Snyder JT, Worthylake DK, Rossman KL, Betts L, Pruitt WM, Siderovski DP, et al. 2002. Structural basis for the selective activation of Rho GTPases by Dbl exchange factors. Nat Struct Biol 9:468-75. doi: 10.1038/ nsb796.

Sot B, Kötting C, Deaconescu D, Suveyzdis Y, Gerwert K, Wittinghofer A. 2010. Unravelling the mechanism of dual-specificity GAPs. EMBO J 29:1205-14. doi: 10.1038/emboj.2010.20.

Takahashi T, Fournier A, Nakamura F, Wang LH, Murakami Y, Kalb RG, et al. 1999. Plexin-neuropilin-1 complexes form functional semaphorin-3A receptors. Cell 99:59-69. doi: 10.1016/S0092-8674(00)80062-8.

Takamatsu H, Kumanogoh A. 2012. Diverse roles for semaphorin-plexin signaling in the immune system. Trends Immunol 33:127-35. doi: 10.1016/j.it.2012.01.008.

Tamagnone L. 2012. Emerging role of semaphorins as major regulatory signals and potential therapeutic targets in cancer. Cancer Cell 22:145-52. doi: 10.1016/j.ccr.2012.06.031.

Tong Y, Chugha P, Hota PK, Alviani RS, Li M, Tempel W, et al. 2007. Binding of Rac1, Rnd1, and RhoD to a novel Rho GTPase interaction motif destabilizes dimerization of the plexin-B1 effector domain. J Biol Chem 282:37215-24. doi: 10.1074/jbc.M703800200.

Tong Y, Hota PK, Penachioni JY, Hamaneh MB, Kim S, Alviani RS, et al. 2009. Structure and function of the intracellular region of the plexin-b1 transmembrane receptor. J Biol Chem 284:35962-72. doi: 10.1074/jbc.M109.056275.

Toyofuku T, Yoshida J, Sugimoto T, Zhang H, Kumanogoh A, Hori M, et al. 2005. FARP2 triggers signals for Sema3Amediated axonal repulsion. Nat Neurosci 8:1712-9. doi: 10.1038/nn1596.

Tran TS, Kolodkin AL, Bharadwaj R. 2007. Semaphorin regulation of cellular morphology. Ann Rev Cell Dev Biol 23:263-92. doi: 10.1146/annurev.cellbio.22.010605.093554.

Turner LJ, Nicholls S, Hall A. 2004. The activity of the plexin-A1 receptor is regulated by Rac. J Biol Chem 279:33199-205. doi: 10.1074/jbc.M402943200.

Vetter IR, Wittinghofer A. 2001. The guanine nucleotide-binding switch in three dimensions. Science 294:1299-304. doi: 10.1126/science.1062023.

Vikis HG, Li W, He Z, Guan KL. 2000. The semaphorin receptor plexin-B1 specifically interacts with active Rac in a ligand-dependent manner. Proc Natl Acad Sci USA 97:12457-62. doi: 10.1073/pnas.220421797.

Wang Y, He H, Srivastava N, Vikarunnessa S, Chen YB, Jiang J, et al. 2012. Plexins are GTPase-activating proteins for Rap and are activated by induced dimerization. Sci Signal 5:ra6. doi: 10.1126/scisignal.2002636.

Webb MR, Hunter JL. 1992. Interaction of GTPase-activating protein with p21ras, measured using a continuous assay for inorganic phosphate release. Biochem J 287(Pt 2):555-9.

Winn MD, Ballard CC, Cowtan KD, Dodson EJ, Emsley P, Evans PR, et al. 2011. Overview of the CCP4 suite and current developments. Acta Crystallogr 67:235-42. doi: 10.1107/S0907444910045749. 
Yaron A, Zheng B. 2007. Navigating their way to the clinic: emerging roles for axon guidance molecules in neurological disorders and injury. Dev Neurobiol 67:1216-31. doi: 10.1002/dneu.20512.

Yatani A, Quilliam LA, Brown AM, Bokoch GM. 1991. Rap1A antagonizes the ability of Ras and Ras-Gap to inhibit muscarinic K+ channels. J Biol Chem 266:22222-6.

Yazdani U, Terman JR. 2006. The semaphorins. Genome Biol 7:211. doi: 10.1186/gb-2006-7-3-211.

Zanata SM, Hovatta I, Rohm B, Püschel AW. 2002. Antagonistic effects of Rnd1 and RhoD GTPases regulate receptor activity in Semaphorin 3A-induced cytoskeletal collapse. J Neurosci 22:471-7.

Zhao H, Ghirlando R, Piszczek G, Curth U, Brautigam CA, Schuck P. 2013. Recorded scan times can limit the accuracy of sedimentation coefficients in analytical ultracentrifugation. Anal Biochem 437:104-8. doi: 10.1016/j.ab.2013.02.011. 